\title{
Numerical wave propagation on the hexagonal C-grid
}

\author{
J. Thuburn ${ }^{\mathrm{a}, *}$, \\ ${ }^{a}$ Mathematics Research Institute, School of Engineering, Computing and \\ Mathematics, University of Exeter, Exeter, EX4 4QE, UK
}

\begin{abstract}
Inertio-gravity mode and Rossby mode dispersion properties are examined for discretizations of the linearized rotating shallow water equations on a regular hexagonal C-grid in planar geometry. It is shown that spurious non-zero Rossby mode frequencies found by previous authors in the $f$-plane case can be avoided by an appropriate discretization of the Coriolis terms. Three generalizations of this discretization that conserve energy even for non-constant Coriolis parameter are presented. A quasigeostrophic $\beta$-plane analysis is carried out to investigate the Rossby mode dispersion properties of these three schemes. The Rossby mode dispersion relation is found to have two branches. The primary branch modes are good approximations, in terms of both structure and frequency, to corresponding modes of the continuous governing equations, and offer some improvements over a quadrilateral C-grid scheme. The secondary branch modes have vorticity structures approximating those of smallscale modes of the continuous governing equations, suggesting that the hexagonal C-grid might have an advantage in terms of resolving extra Rossby modes; however, the frequencies of the secondary branch Rossby modes are much smaller than those of the corresponding continuous modes, so this potential advantage is not fully realized.
\end{abstract}

\section{Introduction}

Geodesic grids, obtained by iterative refinement of a parent icosahedron, are potentially attractive for the horizontal discretization of global atmospheric models because they provide a nearly homogeneous and isotropic coverage of the sphere $[27,19,3,12,9,22,21,8,18,2,13]$. Several groups are now using such grids for complex weather and climate models $[17,11,20]$. There are essentially

\footnotetext{
* Corresponding author

Email address: j.thuburn@ex.ac.uk (J. Thuburn ).
} 
two types of geodesic grid, based either on a triangular refinement of the icosahedron, or on its dual comprised of hexagons and (always exactly 12) pentagons.

Despite growing interest, the theoretical understanding of numerical methods on geodesic grids is rather less developed than it is for Cartesian or longitudelatitude quadrilateral grids. One set of issues involves the numerical wave dispersion properties and how accurately they capture the wave dispersion properties of the continuous governing equations. For quadrilateral grids, a C-grid staggering, in which the mass variable is stored at cell centres and the normal component of the velocity is stored at cells edges, captures inertiogravity wave propagation and hence geostrophic adjustment well provided the Rossby radius is well resolved $[1,16,6]$; an analogous C-grid staggering might be expected to work well on triangular or hexagonal grids. However, the ratio of number of degrees of freedom in the wind field to number of degrees of freedom in the mass field depends on the grid structure, leading to concerns that non-quadrilateral grids might give rise to computational modes that do not propagate or are unphysical in some other way [10].

Ničković et al. [14] analysed the wave dispersion properties under the $f$-plane approximation (that is, assuming planar geometry and constant Coriolis parameter $f$ ) for the linearized shallow water equations on hexagonal grids with several alternative staggerings. For a C-grid staggering they found a numerical dispersion relation with four branches, two corresponding to eastward and westward inertio-gravity modes, and two corresponding to geostrophic or Rossby modes. This may be contrasted with the quadrilateral grid numerical dispersion relation, which has only three branches, corresponding to eastward and westward inertio-gravity modes and a single Rossby mode branch. The extra Rossby mode branch found for the hexagonal grid might be considered an advantage, given that in most practical applications the Rossby modes are of greater interest than the inertio-gravity modes. However, it is necessary to check that the extra Rossby modes really are approximations to physical Rossby modes and not unphysical artefacts of the discretization. In fact both Rossby mode branches found by [14] have the undesirable property of having non-zero frequency, whereas the $f$-plane Rossby mode frequency for the continuous equations is identically zero. Thus the hexagonal C-grid would seem to have a serious drawback in terms of its wave propagation characteristics.

This drawback can be overcome by using a 'skewed' variant of the C-grid with fewer velocity degrees of freedom $[15,14]$, but at the price of sacrificing the isotropy of the grid. The purpose of the present paper is to revisit numerical wave propagation on the standard, isotropic, hexagonal C-grid. On a C-grid of any structure, some spatial averaging is unavoidable in the Coriolis terms; however, there is some choice available both in the stencil for the averaging and in the evaluation and weighting of the Coriolis parameter, and numerical 
Rossby mode propagation can be sensitive to these details [24,25,4,23]. In section 2 it will be shown that an appropriate discretization of the Coriolis terms can remove the unphysical non-zero Rossby mode frequencies on the $f$-plane hexagonal C-grid found by [14]. In section 3, possible generalizations of this discretization to the case of non-constant $f$ are considered. Three alternatives that conserve energy are presented. In section 4 a quasigeostrophic $\beta$-plane analysis is carried out to obtain the Rossby mode dispersion relations for these three energy-conserving schemes. These theoretical dispersion relations are compared with direct numerical calculations of normal mode frequencies in section 5. Section 6 discusses in some detail the extra Rossby mode branch and the extent to which these modes approximate physical solutions of the continous governing equations.

\section{$2 f$-plane dispersion relation}

It is convenient to work in terms of coordinate directions and velocity components normal to the edges of the hexagonal grid. Let

$$
x_{1}=x, \quad x_{2}=\frac{\sqrt{3}}{2} y-\frac{1}{2} x, \quad x_{3}=-\frac{\sqrt{3}}{2} y-\frac{1}{2} x
$$

where $x$ and $y$ are the usual Cartesian coordinates. Let $\hat{\mathbf{x}}_{1}=\nabla x_{1}, \hat{\mathbf{x}}_{2}=\nabla x_{2}$, $\hat{\mathbf{x}}_{3}=\nabla x_{3}$ be the corresponding unit vectors, and let

$$
u_{j}=\mathbf{u} \cdot \hat{\mathbf{x}}_{j}, \quad j=1,2,3
$$

be the velocity components in these directions (see Fig. 1). An important identity in this framework is

$$
\hat{\mathbf{x}}_{1}+\hat{\mathbf{x}}_{2}+\hat{\mathbf{x}}_{3}=0
$$

implying, among other things, that

$$
\partial_{x_{1}} \psi+\partial_{x_{2}} \psi+\partial_{x_{3}} \psi=0
$$

where $\partial_{x_{j}}=\hat{\mathbf{x}}_{j} . \nabla$. (Here, and later, $\psi$ will stand for an arbitrary field either in the continuous equations or on the grid.)

The continuous shallow water equations, linearized about a resting state with mean geopotential $\Phi_{0}$, then become [14]

$$
\partial_{t} \Phi+\frac{2}{3} \Phi_{0}\left(\partial_{x_{1}} u_{1}+\partial_{x_{2}} u_{2}+\partial_{x_{3}} u_{3}\right)=0
$$




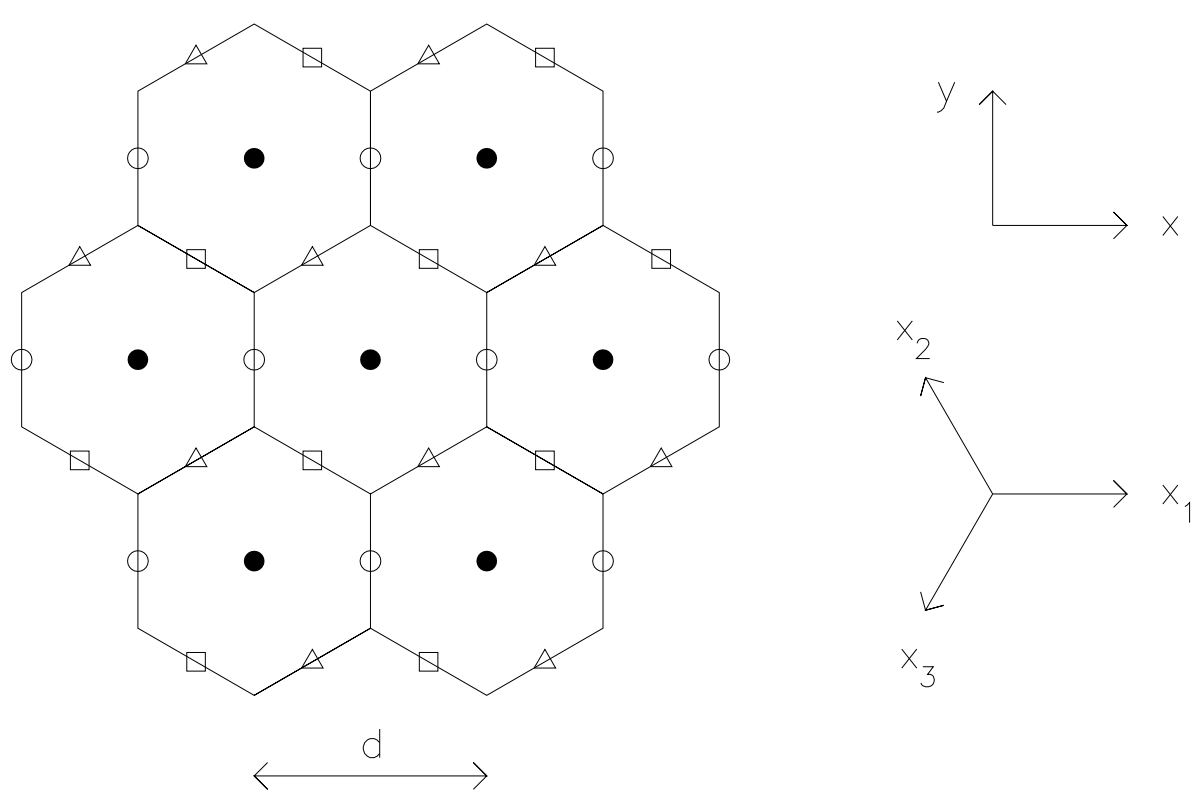

Fig. 1. Schematic showing the hexagonal C-grid configuration and the coordinate systems used in this paper. Filled circles indicate the locations of the $\Phi$ points, open circles $u_{1}$ points, triangles $u_{2}$ points, and squares $u_{3}$ points.

$$
\begin{aligned}
& \partial_{t} u_{1}-\frac{f}{\sqrt{3}}\left(u_{2}-u_{3}\right)+\partial_{x_{1}} \Phi=0, \\
& \partial_{t} u_{2}-\frac{f}{\sqrt{3}}\left(u_{3}-u_{1}\right)+\partial_{x_{2}} \Phi=0, \\
& \partial_{t} u_{3}-\frac{f}{\sqrt{3}}\left(u_{1}-u_{2}\right)+\partial_{x_{3}} \Phi=0,
\end{aligned}
$$

where $\Phi$ is the geopotential perturbation. In this section $f$ will be taken to be a constant $f_{0}$; in later sections a spatial variation of $f$ will be allowed.

A couple of symmetry properties of these equations are worth noting. First, equations (7) and (8) can be obtained from (6) by cyclic permutations of the indices $(1,2,3)$. Second, in (6), terms involving $u_{2}$ appear with opposite sign to terms involving $u_{3}$, etc. Analogous properties hold throughout all of the derivations below. These provide valuable checks on the somewhat lengthy algebra.

These equations have an extra degree of freedom compared with their analogues in Cartesian coordinates. We should therefore expect them to satisfy some constraint, otherwise they might support spurious solutions associated with the extra degree of freedom. From the definition of the velocity components and unit vectors

$$
u_{1}+u_{2}+u_{3}=\mathbf{u} .\left(\hat{\mathbf{x}}_{1}+\hat{\mathbf{x}}_{2}+\hat{\mathbf{x}}_{3}\right)=0 .
$$


Adding (6), (7) and (8) shows that

$$
\partial_{t}\left(u_{1}+u_{2}+u_{3}\right)=0
$$

so that the constraint (9) is maintained provided it is satisfied initially.

The governing equations are now discretized in space on a hexagonal C-grid; see Fig. 1. The distance between neighbouring $\Phi$ points is $d$. To begin with we review the analysis for the scheme discussed by [14]:

$$
\begin{aligned}
& \partial_{t} \Phi+\frac{2}{3} \Phi_{0}\left(\delta_{1} u_{1}+\delta_{2} u_{2}+\delta_{3} u_{3}\right)=0 \\
& \partial_{t} u_{1}-\frac{f_{0}}{\sqrt{3}}\left({\overline{u_{2}}}^{3}-{\overline{u_{3}}}^{2}\right)+\delta_{1} \Phi=0 \\
& \partial_{t} u_{2}-\frac{f_{0}}{\sqrt{3}}\left({\overline{u_{3}}}^{1}-{\overline{u_{1}}}^{3}\right)+\delta_{2} \Phi=0 \\
& \partial_{t} u_{3}-\frac{f_{0}}{\sqrt{3}}\left({\overline{u_{1}}}^{2}-{\overline{u_{2}}}^{1}\right)+\delta_{3} \Phi=0
\end{aligned}
$$

Here, $\delta_{j}$ is the centred, second-order, two-point finite difference approximation to $\partial_{x_{j}}$, and $\overline{(.)}^{j}$ is the two-point average in the $x_{j}$ direction.

If the domain is periodic or infinite in each direction then the discrete equations will support solutions proportional to $\exp \{\mathrm{i}(\mathbf{k} \cdot \mathbf{x}-\omega t\}$, where $\mathbf{k}=(k, l)$ is the wave vector and $\omega$ is the frequency. For such solutions, we may replace $\partial_{t}$ by $-\mathrm{i} \omega,{\overline{u_{i}}}^{j}$ by $c_{j} u_{i}$ and $\delta_{j} \Phi$ by $2 \mathrm{i} s_{j} \Phi / d$ in (11)-(14), where

$$
\begin{gathered}
c_{j}=\cos \left(k_{j} d / 2\right), \\
s_{j}=\sin \left(k_{j} d / 2\right),
\end{gathered}
$$

with $k_{j}=\mathbf{k} . \hat{\mathbf{x}}_{j}$. Thus,

$$
\begin{aligned}
& -\mathrm{i} \omega \Phi+\frac{4 \mathrm{i}}{3 d} \Phi_{0}\left(u_{1} s_{1}+u_{2} s_{2}+u_{3} s_{3}\right)=0 \\
& -\mathrm{i} \omega u_{1}-\frac{f_{0}}{\sqrt{3}}\left(u_{2} c_{3}-u_{3} c_{2}\right)+\frac{2 \mathrm{i} s_{1}}{d} \Phi=0, \\
& -\mathrm{i} \omega u_{2}-\frac{f_{0}}{\sqrt{3}}\left(u_{3} c_{1}-u_{1} c_{3}\right)+\frac{2 \mathrm{i} s_{2}}{d} \Phi=0, \\
& -\mathrm{i} \omega u_{3}-\frac{f_{0}}{\sqrt{3}}\left(u_{1} c_{2}-u_{2} c_{1}\right)+\frac{2 \mathrm{i} s_{3}}{d} \Phi=0 .
\end{aligned}
$$


Then, eliminating $u_{1}, u_{2}$ and $u_{3}$ and finally cancelling $\Phi$ (or, equivalently, demanding that the determinant of this $4 \times 4$ linear system should vanish) leads to the dispersion relation for the discrete equations

$$
\begin{aligned}
\omega^{4}-\omega^{2}\left\{\frac { f _ { 0 } ^ { 2 } } { 3 } \left(c_{1}^{2}+c_{2}^{2}\right.\right. & \left.\left.+c_{3}^{2}\right)+\frac{8 \Phi_{0}}{3 d^{2}}\left(s_{1}^{2}+s_{2}^{2}+s_{3}^{2}\right)\right\} \\
& +\frac{8 \Phi_{0} f_{0}^{2}}{9 d^{2}}\left(s_{1} c_{1}+s_{2} c_{2}+s_{3} c_{3}\right)^{2}=0
\end{aligned}
$$

As discussed by [14], this has four roots, two corresponding to eastward and westward propagating inertio-gravity modes, and two corresponding to "geostrophic" modes. In the limit of small wavenumber $|\mathbf{k}| d \ll 1$ we have $c_{j} \sim 1$, $2 s_{j} / d \sim k_{j},\left(8 / 3 d^{2}\right)\left(s_{1}^{2}+s_{2}^{2}+s_{3}^{2}\right) \sim|\mathbf{k}|^{2}$, and $s_{1} c_{1}+s_{2} c_{2}+s_{3} c_{3} \sim 0$. Then $(21)$ becomes

$$
\omega^{4}-\omega^{2}\left\{f_{0}^{2}+\Phi_{0}|\mathbf{k}|^{2}\right\} \approx 0
$$

giving the correct frequencies for inertio-gravity modes

$$
\omega^{2} \approx f_{0}^{2}+\Phi_{0}|\mathbf{k}|^{2}
$$

and the geostrophic modes $(\omega \approx 0)$. For finite $|\mathbf{k}| d$ the factor $\left(c_{1}^{2}+c_{2}^{2}+c_{3}^{2}\right) / 3$ is reduced below 1 , leading to a reduction of the Coriolis effect on inertiogravity modes that is well known for the quadrilateral C-grid. More importantly, however, the last term in (21) does not generally vanish, so the geostrophic modes must have non-zero frequency. Moreover, as shown by [14], this frequency is not small but can be of order $f_{0}$.

For a quadrilateral C-grid, [23] found that improved Rossby mode behaviour could be obtained by averaging $u$ to $\Phi$ points before picking up the Coriolis factor and averaging to $v$ points, and averaging $v$ to $\Phi$ points before picking up the Coriolis factor and averaging to $u$ points. Seeking a hexagonal grid analogue of this $f$-at- $\Phi$-point scheme suggests an alternative to the scheme analysed by [14] in which the contributions to the tangential velocity at each cell edge are obtained by averaging over four neighbours rather than two:

$$
\begin{aligned}
& \partial_{t} u_{1}-\frac{f_{0}}{\sqrt{3}}\left({\overline{u_{2}}}^{2}-{\overline{u_{3}}}^{31}\right)+\delta_{1} \Phi=0 \\
& \partial_{t} u_{2}-\frac{f_{0}}{\sqrt{3}}\left({\overline{u_{3}}}^{32}-{\overline{u_{1}}}^{12}\right)+\delta_{2} \Phi=0 \\
& \partial_{t} u_{3}-\frac{f_{0}}{\sqrt{3}}\left({\overline{u_{1}}}^{13}-{\overline{u_{2}}}^{23}\right)+\delta_{3} \Phi=0 .
\end{aligned}
$$


Here $\bar{\psi}^{i j}$ is shorthand for $\overline{\bar{\psi}}^{j}$. (Note, also, that averages in different directions commute with each other and also with finite difference derivatives.) The $\Phi$ equation (11) remains unchanged.

The dispersion analysis proceeds exactly as for the previous scheme, except that $c_{1}$ is replaced by $c_{2} c_{3}, c_{2}$ by $c_{3} c_{1}$, and $c_{3}$ by $c_{1} c_{2}$ throughout. Unfortunately the resulting dispersion relation still has a non-zero coefficient of $\omega^{0}$ so the scheme still supports geostrophic modes of non-zero frequency.

A third scheme can be obtained by taking $2 / 3$ times (24)-(26) plus $1 / 3$ times (12)-(14):

$$
\begin{aligned}
& \partial_{t} u_{1}-\frac{f_{0}}{\sqrt{3}}\left({\widetilde{u_{2}}}^{3}-{\widetilde{u_{3}}}^{2}\right)+\delta_{1} \Phi=0, \\
& \partial_{t} u_{2}-\frac{f_{0}}{\sqrt{3}}\left({\widetilde{u_{3}}}^{1}-{\widetilde{u_{1}}}^{3}\right)+\delta_{2} \Phi=0, \\
& \partial_{t} u_{3}-\frac{f_{0}}{\sqrt{3}}\left({\widetilde{u_{1}}}^{2}-{\widetilde{u_{2}}}^{1}\right)+\delta_{3} \Phi=0 .
\end{aligned}
$$

The notation $\widetilde{\psi}^{1}$ to stand for $\left(2 \bar{\psi}^{23}+\bar{\psi}^{1}\right) / 3$, along with the obvious permutations, will be useful throughout the rest of this paper. Again the $\Phi$ equation (11) remains unchanged.

It can be shown that this new averaging operator satisfies the following identity:

$$
\delta_{1} \widetilde{\psi}^{1}+\delta_{2} \widetilde{\psi}^{2}+\delta_{3} \widetilde{\psi}^{3}=0
$$

This is the analogue of the continuous equation identity (4), suggesting that $\widetilde{(.)}$ is a natural averaging operator on the hexagonal C-grid.

Now the dispersion analysis proceeds exactly as for the first scheme except that $c_{1}$ is replaced by $a_{1}=\left(2 c_{2} c_{3}+c_{1}\right) / 3, c_{2}$ by $a_{2}=\left(2 c_{3} c_{1}+c_{2}\right) / 3$, and $c_{3}$ by $a_{3}=\left(2 c_{1} c_{2}+c_{3}\right) / 3$ throughout. In this case the coefficient of $\omega^{0}$ is found to vanish on account of the identity

$$
s_{1} a_{1}+s_{2} a_{2}+s_{3} a_{3}=0
$$

which can be derived using standard trigonometric identities starting from the fact that $k_{1}+k_{2}+k_{3}=0$, or by substituting wavelike solutions into (30). 
The resulting dispersion relation is

$$
\omega^{4}-\omega^{2}\left\{\frac{f_{0}^{2}}{3}\left(a_{1}^{2}+a_{2}^{2}+a_{3}^{2}\right)+\frac{8 \Phi_{0}}{3 d^{2}}\left(s_{1}^{2}+s_{2}^{2}+s_{3}^{2}\right)\right\}=0 .
$$

As for the previous schemes, this gives the correct dispersion relation in the limit $|\mathbf{k}| d \ll 1$, but, unlike them, it always gives frequency exactly zero for the geostrophic modes.

Figure 2 shows the exact inertio-gravity mode dispersion relation and the corresponding numerical inertio-gravity mode dispersion relations for the scheme analysed by [14] and the new scheme (27)-(29) (with (11)) analysed here. The parameters have been chosen to give a well-resolved Rossby radius. The numerical dispersion relations are very nearly isotropic, and the two numerical schemes give very similar results. Wave frequencies are well approximated for small wavenumbers but are artificially slowed as the finite difference derivatives become less accurate for large wavenumbers. Also shown is the dispersion relation for the geostrophic modes for the scheme analysed by [14]. For the shortest resolved wavelengths the frequencies are close to $f_{0} / 2$.

Figure 3 shows analogous results for a case with poorly resolved Rossby radius. Both numerical schemes accurately capture the inertio-gravity mode frequencies for small wavenumbers, but give an unrealistic decrease of frequency with increasing wavenumber due to the averaging of the Coriolis terms, which dominate pressure gradients in this parameter regime. This decrease is worse for the new scheme, consistent with the larger stencil used to average the Coriolis terms. In this regime too, the geostrophic modes of the [14] scheme have maximum frequency close to $f_{0} / 2$.

\section{Energy conserving generalizations of the Coriolis term discretiza- tion}

The Coriolis force acts normal to the flow and therefore does no work, so the Coriolis terms conserve energy. It is highly desirable that a numerical scheme should retain an analogous property. On an $f$-plane the Coriolis terms in (27)(29) do have this property, in other words, if we take the sum over all grid points of $u_{1}$ times (27) plus $u_{2}$ times (28) plus $u_{3}$ times (29) to form a kinetic energy equation we find that all Coriolis terms cancel.

We wish to extend this result to allow for arbitrary spatial variations in $f$; we do not restrict attention to the case $f=f(y)$ because we wish to consider arbitrary orientations of the grid relative to the northward direction. We have some freedom in whether we choose to multiply by $f$ before averaging (e.g. 

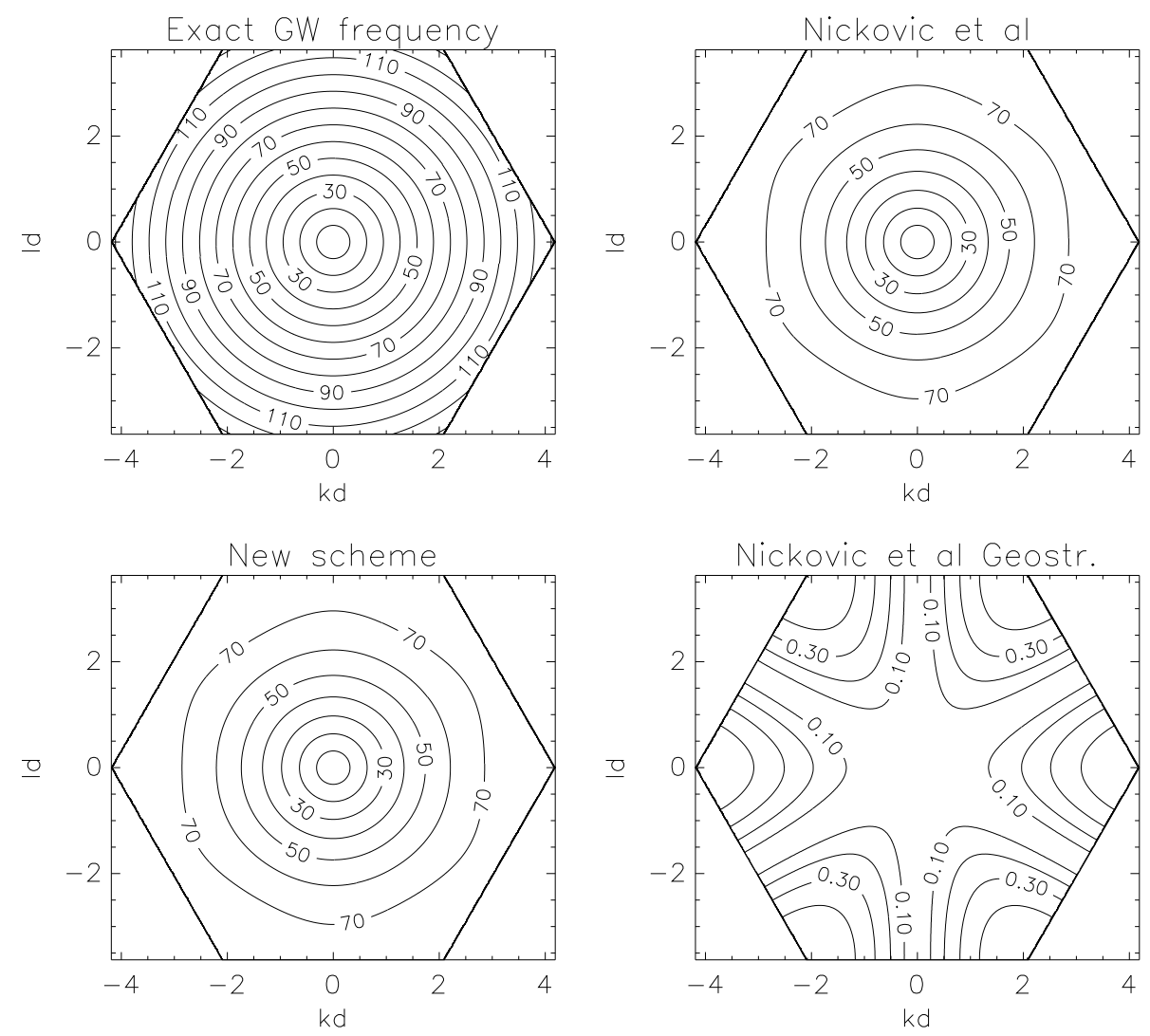

Fig. 2. Values of $\omega / f_{0}$ versus $k d$ and $l d$ for continuous and discrete dispersion relations. Top left: inertio-gravity modes for the continuous equations. Top right: inertio-gravity modes for the scheme analysed by [14]. Bottom left: inertio-gravity modes for the new scheme analysed here. Bottom right: geostrophic modes for the scheme analysed by [14]. The hexagonal region shows the range of resolvable wavenumbers on the hexagonal grid. The parameters used are $d=10^{5} \mathrm{~m}, \Phi_{0}=10^{5} \mathrm{~m}^{2} \mathrm{~s}^{-2}$, and $f_{0}=10^{-4} \mathrm{~s}^{-1}$, implying a resolution factor $d / \lambda=0.0316$ where $\lambda=\sqrt{\Phi_{0}} / f_{0}$ is the Rossby radius.

${\widetilde{f u_{2}}}^{3}$ ), after averaging (e.g. $f{\widetilde{u_{2}}}^{3}$ ), or at an intermediate stage in the averaging process; these options are all equivalent when $f$ is constant, but not when it varies.

We require that all terms proportional to $u_{1} u_{2}$ that arise from $u_{1}$ times the $u_{1}$ equation should cancel with corresponding terms from $u_{2}$ times the $u_{2}$ equation, etc. By considering the symmetries of the terms involved, the following three schemes are seen to conserve energy without introducing a preferred direction in the discretization:

Scheme (i): $f u_{2}$ at $u_{1}$ points is discretized as $\left(4{\overline{f{\overline{u_{2}}}^{2}}}^{1}+f{\overline{u_{2}}}^{3}+{\overline{f u_{2}}}^{3}\right) / 6$, etc;

Scheme (ii): $f u_{2}$ at $u_{1}$ points is discretized as $\left(f{\widetilde{u_{2}}}^{3}+{\widetilde{f u_{2}}}^{3}\right) / 2$, etc. 

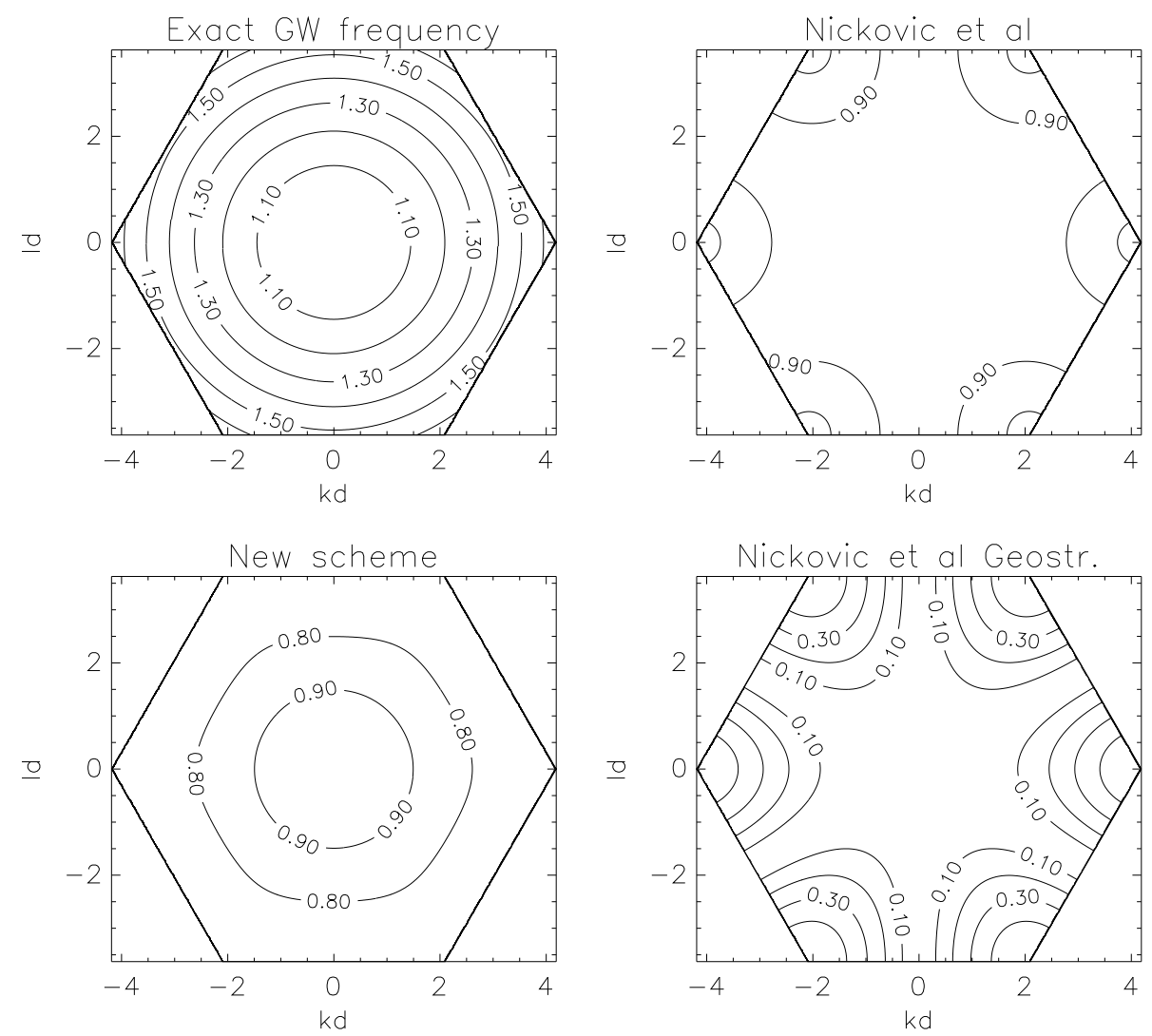

Fig. 3. As in Fig. 2 except that $\Phi_{0}$ is reduced to $10 \mathrm{~m}^{2} \mathrm{~s}^{-2}$, implying $d / \lambda=3.16$.

Scheme (iii): $f u_{2}$ at $u_{1}$ points is discretized as $\left(4{\overline{f{\overline{u_{2}}}^{1}}}^{2}+f{\overline{u_{2}}}^{3}+{\overline{f u_{2}}}^{3}\right) / 6$, etc;

(We could also take any linear combination of these three). Scheme (i) is the most similar in form to the $f$-at- $\Phi$-points discretization discussed by [23], since the $f$ in its first term is indeed evaluated at a $\Phi$ point.

Finally, for completeness, note that the other terms in the spatial discretization are also energy conserving: upon taking $2 / 3$ of $\Phi_{0} u_{1}$ times (27) plus $\Phi_{0} u_{2}$ times (28) plus $\Phi_{0} u_{3}$ times (29) and adding to $\Phi$ times (11) and summing over all grid cells, we find that all contributions from terms involving $\Phi$ times $u_{j}$ cancel, implying zero tendency of the total energy.

\section{Quasigeostrophic $\beta$-plane dispersion relation}

In order to understand the properties of the two branches of geostrophic or Rossby modes, and determine whether they are approximations to physical modes or merely numerical artefacts, it is necessary to compare them with Rossby mode solutions of the continuous equations when there is a $\beta$-effect, i.e. a spatial variation in $f$, so that the Rossby modes are non-degenerate. 
However, if $f$ is allowed to vary then (5)-(8) no longer support exact wavelike solutions proportional to $\exp \{\mathrm{i}(\mathbf{k} \cdot \mathbf{x}-\omega t\}$. Progress can be made, in both the continuous and discrete cases, by making the quasigeostrophic $\beta$-plane approximation $[26,5,7]$.

On the hexagonal C-grid there are some additional mathematical complications and subtleties linked to the extra degree of freedom and the associated extra Rossby mode branch. The derivation is therefore given below in some detail. We consider the three energy conserving schemes proposed in section 3, starting with scheme (i).

\subsection{Scheme (i)}

For scheme (i) the spatially discretized momentum equations become

$$
\begin{aligned}
& \partial_{t} u_{1}-\frac{1}{6 \sqrt{3}}\left\{\left(4{\overline{f \bar{u}^{2}}}^{1}+f{\overline{u_{2}}}^{3}+{\overline{f u_{2}}}^{3}\right)-\left(4{\overline{f \bar{u}_{3}}}^{1}+f{\overline{u_{3}}}^{2}+{\overline{f u_{3}}}^{2}\right)\right\} \\
& +\delta_{1} \Phi=0 \\
& \partial_{t} u_{2}-\frac{1}{6 \sqrt{3}}\left\{\left(4{\overline{f \bar{u}^{3}}}^{2}+f{\overline{u_{3}}}^{1}+{\overline{f u_{3}}}^{1}\right)-\left(4{\overline{f \bar{u}_{1}}}^{2}+f{\overline{u_{1}}}^{3}+{\overline{f u_{1}}}^{3}\right)\right\} \\
& +\delta_{2} \Phi=0 \\
& \partial_{t} u_{3}-\frac{1}{6 \sqrt{3}}\left\{\left(4{\overline{f \bar{u}_{1}}}^{3}+f{\overline{u_{1}}}^{2}+{\overline{f u_{1}}}^{2}\right)-\left(4{\overline{f \bar{u}^{2}}}^{3}+f{\overline{u_{2}}}^{1}+{\overline{f u_{2}}}^{1}\right)\right\} \\
& +\delta_{3} \Phi=0 \text {. }
\end{aligned}
$$

Now let $f=f_{0}+f^{\prime}$, where $f_{0}$ is constant and $f^{\prime}=\boldsymbol{\beta} . \mathbf{x}$ has a constant spatial gradient $\boldsymbol{\beta}=(\alpha, \beta)$. (Retaining both components of $\boldsymbol{\beta}$ will allow us to investigate the effects of changing the alignment of the grid relative to the northward direction.) Assume that variations in $f^{\prime}$ on the spatial scale of interest $L$ are much smaller than $f_{0}$, i.e. $L|\boldsymbol{\beta}| \ll f_{0}$, and that the flow evolves on a timescale much longer than $1 / f_{0}$, i.e. $\partial_{t} \ll f_{0}$. Together with the assumption $|\Phi| \ll \Phi_{0}$, which we already made in linearizing the equations, these are the standard assumptions for quasigeostrophic $\beta$-plane shallow water theory.

Under these assumptions the leading terms in (33)-(35) define the geostrophic 
velocity. We will write

$$
u_{j}=u_{g j}+u_{a j}
$$

where $u_{g j}$ is the geostrophic component and $u_{a j}$ is the ageostrophic component, but then drop the subscript $g$ in what follows to reduce the clutter of symbols. The leading terms in (33)-(35) are then

$$
\begin{aligned}
-\frac{f_{0}}{\sqrt{3}}\left({\widetilde{u^{2}}}^{3}-{\widetilde{u_{3}}}^{2}\right)+\delta_{1} \Phi & =0, \\
-\frac{f_{0}}{\sqrt{3}}\left({\widetilde{u_{3}}}^{1}-{\widetilde{u_{1}}}^{3}\right)+\delta_{2} \Phi & =0, \\
-\frac{f_{0}}{\sqrt{3}}\left({\widetilde{u_{1}}}^{2}-{\widetilde{u_{2}}}^{1}\right)+\delta_{3} \Phi & =0 .
\end{aligned}
$$

An important point is that this system, considered as a set of equations for $u_{1}$, $u_{2}$ and $u_{3}$ in terms of $\Phi$, is singular and so has a solution only if a solvability condition is satisfied. The solvability condition can be found by applying $\widetilde{(.)^{1}}$ to $(37), \widetilde{(.)}^{2}$ to $(38)$, and $\widetilde{(.)}^{3}$ to $(39)$, and summing to obtain

$$
\delta_{1} \widetilde{\Phi}^{1}+\delta_{2} \widetilde{\Phi}^{2}+\delta_{3} \widetilde{\Phi}^{3}=0 .
$$

By (30), this is indeed satisfied, so there is a solution for the geostrophic flow. Note that the use of the averaging operator $\widetilde{(.)}$ as the basis for the discretization of the Coriolis terms is crucial here. The solvability condition arising with other averaging operators would not be satisfied. This is consistent with the existence of geostrophic mode frequencies of order $f_{0}$ so that the quasigeostrophic assumption $\partial_{t} \ll f_{0}$ is not satisfied for other averaging operators.

Because the system (37)-(39) is singular, its solution (if it exists) is nonunique. For future reference, we note that, for wavelike solutions proportional to $\exp \{\mathrm{i}(\mathbf{k} \cdot \mathbf{x}-\omega t)\}$, the solution is

$$
\left(\begin{array}{l}
u_{1} \\
u_{2} \\
u_{3}
\end{array}\right)=\frac{2 \mathrm{i}}{f_{0} d \sqrt{3}}\left\{\left(\begin{array}{c}
-p_{1} \\
-p_{2} \\
-p_{3}
\end{array}\right) \Phi+\left(\begin{array}{l}
a_{1} \\
a_{2} \\
a_{3}
\end{array}\right) \mu\right\},
$$

where 


$$
\begin{aligned}
& p_{1}=s_{2} / a_{3}-s_{3} / a_{2}, \\
& p_{2}=s_{3} / a_{1}-s_{1} / a_{3}, \\
& p_{3}=s_{1} / a_{2}-s_{2} / a_{1},
\end{aligned}
$$

and $\mu$ is an arbitrary complex number times $\exp \{\mathrm{i}(\mathbf{k} \cdot \mathbf{x}-\omega t)\}$, making explicit the non-uniqueness.

The usual approach in quasigeostrophic theory (both for the continuous equations and for the discrete equations on quadrilateral grids) is to determine the time evolution of the geostrophic flow by going to next order in the momentum equations, forming the vorticity equation, and eliminating the ageostrophic divergence via the mass continuity equation. In the present case we have the additional unknown $\mu$ and so we will require an additional constraint to determine the solution. This extra constraint will come from the solvability condition for the ageostrophic flow at next order, leading to two coupled equations for the two unknowns $\omega$ and $\mu / \Phi$.

At next order the discrete momentum equations are

$$
\begin{aligned}
& \partial_{t} u_{1}-\frac{f_{0}}{\sqrt{3}}\left({\widetilde{u_{a 2}}}^{3}-{\widetilde{u_{a 3}}}^{2}\right) \\
& -\frac{1}{6 \sqrt{3}}\left\{\left(4 \bar{f}^{\prime{\overline{u_{2}}}^{2}}{ }^{1}+f^{\prime}{\overline{u_{2}}}^{3}+{\overline{f^{\prime} u_{2}}}^{3}\right)\right.
\end{aligned}
$$

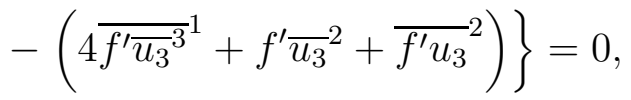

$$
\begin{aligned}
& \partial_{t} u_{2}-\frac{f_{0}}{\sqrt{3}}\left({\widetilde{u_{a 3}}}^{1}-{\widetilde{u_{a 1}}}^{3}\right) \\
& -\frac{1}{6 \sqrt{3}}\left\{\left(4 \bar{f}^{\prime}{\overline{u_{3}}}^{2}+f^{\prime}{\overline{u_{3}}}^{1}+{\overline{f^{\prime} u_{3}}}^{1}\right)\right. \\
& \left.-\left(4 \bar{f}^{\prime}{\overline{u_{1}}}^{2}+f^{\prime}{\overline{u_{1}}}^{3}+{\overline{f^{\prime} u_{1}}}^{3}\right)\right\}=0, \\
& \partial_{t} u_{3}-\frac{f_{0}}{\sqrt{3}}\left({\widetilde{u_{a 1}}}^{2}-{\widetilde{u_{a 2}}}^{1}\right) \\
& -\frac{1}{6 \sqrt{3}}\left\{\left(4 \bar{f}^{\prime}{\overline{u_{1}}}^{3}+f^{\prime}{\overline{u_{1}}}^{2}+{\overline{f^{\prime} u_{1}}}^{2}\right)\right.
\end{aligned}
$$

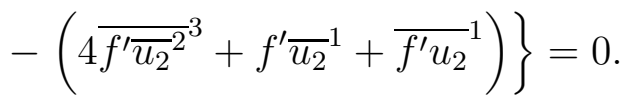


By repeated application in different directions of the one-dimensional identity

$$
\overline{a b}=\bar{a} \bar{b}+\frac{1}{4} \Delta a \Delta b
$$

for any two fields $a$ and $b$, where overbar indicates a two point average and $\Delta$ indicates a two-point difference, and making use of the fact that second differences of $f^{\prime}$ vanish because its gradient is constant, we can rewrite the terms involving $f^{\prime}$. For example,

$$
4{\overline{f^{\prime}{\overline{u_{2}}}^{2}}}^{1}+f^{\prime}{\overline{u_{2}}}^{3}+{\overline{f^{\prime} u_{2}}}^{3}=6 f^{\prime}{\widetilde{u_{2}}}^{3}+d^{2}\left(\beta_{1} \delta_{1}{\overline{u_{2}}}^{2}+\frac{1}{4} \beta_{3} \delta_{3} u_{2}\right),
$$

etc., where $\beta_{j}=\delta_{j} f^{\prime}=\hat{\mathbf{x}}_{j} . \nabla f^{\prime}$. Equations (45)-(47) then become

$$
\begin{aligned}
& \partial_{t} u_{1}-\frac{f_{0}}{\sqrt{3}}\left({\widetilde{u_{a} 2}}^{3}-{\widetilde{u_{a 3}}}^{2}\right)-\frac{f^{\prime}}{\sqrt{3}}\left({\widetilde{u_{2}}}^{3}-{\widetilde{u_{3}}}^{2}\right)-\frac{d^{2}}{6 \sqrt{3}} B_{1}=0, \\
& \partial_{t} u_{2}-\frac{f_{0}}{\sqrt{3}}\left({\widetilde{u_{a 3}}}^{1}-{\widetilde{u_{a 1}}}^{3}\right)-\frac{f^{\prime}}{\sqrt{3}}\left({\widetilde{u_{3}}}^{1}-{\widetilde{u_{1}}}^{3}\right)-\frac{d^{2}}{6 \sqrt{3}} B_{2}=0, \\
& \partial_{t} u_{3}-\frac{f_{0}}{\sqrt{3}}\left({\widetilde{u_{a 1}}}^{2}-{\widetilde{u_{a} 2}}^{1}\right)-\frac{f^{\prime}}{\sqrt{3}}\left({\widetilde{u_{1}}}^{2}-{\widetilde{u_{2}}}^{1}\right)-\frac{d^{2}}{6 \sqrt{3}} B_{3}=0,
\end{aligned}
$$

where

$$
\begin{aligned}
& B_{1}=\left(\beta_{1} \delta_{1}{\overline{u_{2}}}^{2}+\frac{1}{4} \beta_{3} \delta_{3} u_{2}\right)-\left(\beta_{1} \delta_{1}{\overline{u_{3}}}^{3}+\frac{1}{4} \beta_{2} \delta_{2} u_{3}\right), \\
& B_{2}=\left(\beta_{2} \delta_{2}{\overline{u_{3}}}^{3}+\frac{1}{4} \beta_{1} \delta_{1} u_{3}\right)-\left(\beta_{2} \delta_{2}{\overline{u_{1}}}^{1}+\frac{1}{4} \beta_{3} \delta_{3} u_{1}\right), \\
& B_{3}=\left(\beta_{3} \delta_{3} \bar{u}_{1}^{1}+\frac{1}{4} \beta_{2} \delta_{2} u_{1}\right)-\left(\beta_{3} \delta_{3} \bar{u}^{2}+\frac{1}{4} \beta_{1} \delta_{1} u_{2}\right) .
\end{aligned}
$$

First we find the solvability condition for the ageostrophic flow. Take $\widetilde{(.)}{ }^{1}$ of (50) plus $\widetilde{(.)}^{2}$ of $(51)$ plus $\widetilde{(.)}^{3}$ of (52) to obtain

$$
\begin{aligned}
& \partial_{t}\left({\widetilde{u_{1}}}^{1}+{\widetilde{u_{2}}}^{2}+{\widetilde{u_{3}}}^{3}\right)
\end{aligned}
$$

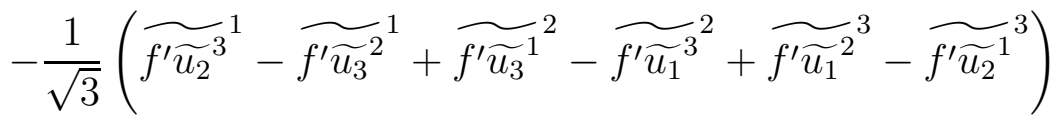

$$
\begin{aligned}
& -\frac{d^{2}}{6 \sqrt{3}}\left({\widetilde{B_{1}}}^{1}+{\widetilde{B_{2}}}^{2}+{\widetilde{B_{3}}}^{3}\right)=0 .
\end{aligned}
$$


Using (48), the terms involving $f^{\prime}$ may be simplified. For example

$$
6{\widetilde{f^{\prime} \psi}}^{1}=6 f^{\prime} \widetilde{\psi}^{1}+d^{2} T_{1}(\psi)
$$

where the operator $T_{1}$ is defined by

$$
T_{1}(\psi)=\left(\beta_{3} \delta_{3} \bar{\psi}^{2}+\beta_{2} \delta_{2} \bar{\psi}^{3}+\frac{1}{2} \beta_{1} \delta_{1} \psi\right)
$$

Analogous relations hold for ${\widetilde{f^{\prime}}}^{2}$ and ${\widetilde{f^{\prime}}}^{3}$ with $T_{2}$ and $T_{3}$ defined by cyclic permutation of indices in (58). The solvability condition (56) becomes

$$
\begin{gathered}
\partial_{t}\left({\widetilde{u_{1}}}^{1}+{\widetilde{u_{2}}}^{2}+{\widetilde{u_{3}}}^{3}\right) \\
-\frac{d^{2}}{6 \sqrt{3}}\left\{T_{1}\left({\widetilde{u_{2}}}^{3}-{\widetilde{u_{3}}}^{2}\right)+T_{2}\left({\widetilde{u_{3}}}^{1}-{\widetilde{u_{1}}}^{3}\right)+T_{3}\left({\widetilde{u_{1}}}^{2}-{\widetilde{u_{2}}}^{1}\right)\right\} \\
-\frac{d^{2}}{6 \sqrt{3}}\left({\widetilde{B_{1}}}^{1}+{\widetilde{B_{2}}}^{2}+{\widetilde{B_{3}}}^{3}\right)=0 .
\end{gathered}
$$

Finally, substitute from (37)-(39) to obtain

$$
\begin{array}{r}
\partial_{t}\left({\widetilde{u_{1}}}^{1}+{\widetilde{u_{2}}}^{2}+{\widetilde{u_{3}}}^{3}\right) \\
-\frac{d^{2}}{6 f_{0}}\left\{T_{1}\left(\delta_{1} \Phi\right)+T_{2}\left(\delta_{2} \Phi\right)+T_{3}\left(\delta_{3} \Phi\right)\right\} \\
\quad-\frac{d^{2}}{6 \sqrt{3}}\left({\widetilde{B_{1}}}^{1}+{\widetilde{B_{2}}}^{2}+{\widetilde{B_{3}}}^{3}\right)=0 .
\end{array}
$$

Now turn to the mass continuity equation:

$$
\partial_{t} \Phi+\frac{2 \Phi_{0}}{3}\left\{\delta_{1}\left(u_{1}+u_{a 1}\right)+\delta_{2}\left(u_{2}+u_{a 2}\right)+\delta_{3}\left(u_{3}+u_{a 3}\right)\right\}=0 .
$$

We must first establish a discrete analogue of the condition that the geostrophic flow is non-divergent. Take $\widetilde{(.)}^{2}$ of (39) minus $\widetilde{(.)}^{3}$ of (38) to obtain

$$
-\frac{f_{0}}{\sqrt{3}}\left({\widetilde{u_{1}}}^{22}-{\widetilde{u_{2}}}^{12}-{\widetilde{u_{3}}}^{13}+{\widetilde{u_{1}}}^{33}\right)+\delta_{3} \widetilde{\Phi}^{2}-\delta_{2} \widetilde{\Phi}^{3}=0
$$

with two further equations obtained by cyclic permutation of indices

$$
-\frac{f_{0}}{\sqrt{3}}\left({\widetilde{u_{2}}}^{33}-{\widetilde{u_{3}}}^{23}-{\widetilde{u_{1}}}^{21}+{\widetilde{u_{2}}}^{11}\right)+\delta_{1} \widetilde{\Phi}^{3}-\delta_{3} \widetilde{\Phi}^{1}=0
$$




$$
-\frac{f_{0}}{\sqrt{3}}\left({\widetilde{u_{3}}}^{11}-{\widetilde{u_{1}}}^{31}-{\widetilde{u_{2}}}^{32}+{\widetilde{u_{3}}}^{22}\right)+\delta_{2} \widetilde{\Phi}^{1}-\delta_{1} \widetilde{\Phi}^{2}=0
$$

Now take $\delta_{1}$ of (62) plus $\delta_{2}$ of (63) plus $\delta_{3}$ of (64); the $\Phi$ terms cancel leaving

$$
\begin{aligned}
& \delta_{1}\left({\widetilde{u_{1}}}^{22}-{\widetilde{u_{2}}}^{12}-{\widetilde{u_{3}}}^{13}+{\widetilde{u_{1}}}^{33}\right)+ \\
& \delta_{2}\left({\widetilde{u_{2}}}^{33}-{\widetilde{u_{3}}}^{23}-{\widetilde{u_{1}}}^{21}+{\widetilde{u_{2}}}^{11}\right)+ \\
& \delta_{3}\left({\widetilde{u_{3}}}^{11}-{\widetilde{u_{1}}}^{31}-{\widetilde{u_{2}}}^{32}+{\widetilde{u_{3}}}^{2}\right)=0 .
\end{aligned}
$$

Then use the identity (30) with $\psi$ equal to ${\widetilde{u_{1}}}^{1},{\widetilde{u_{2}}}^{2}$ and ${\widetilde{u_{3}}}^{3}$ in turn to obtain

$$
\begin{aligned}
\delta_{1}\left({\widetilde{u_{1}}}^{11}+{\widetilde{u_{1}}}^{22}+{\widetilde{u_{1}}}^{33}\right)+ & \delta_{2}\left({\widetilde{u_{2}}}^{11}+{\widetilde{u_{2}}}^{22}+{\widetilde{u_{2}}}^{33}\right)+ \\
& \delta_{3}\left({\widetilde{u_{3}}}^{11}+{\widetilde{u_{3}}}^{22}+{\widetilde{u_{3}}}^{33}\right)=0 .
\end{aligned}
$$

This is the discrete analogue of the condition that the geostrophic flow is nondivergent. We can therefore eliminate the divergence of the geostrophic flow from the mass continuity equation (61) to obtain

$$
\begin{gathered}
\partial_{t}\left(\widetilde{\Phi}^{11}+\widetilde{\Phi}^{22}+\widetilde{\Phi}^{33}\right)+ \\
\frac{2 \Phi_{0}}{3}\left\{\delta_{1}\left({\widetilde{u_{a} 1}}^{11}+{\widetilde{u_{a 1}}}^{2}{ }^{2}+{\widetilde{u_{a 1}}}^{33}\right)+\right. \\
\delta_{2}\left({\widetilde{u_{a} 2}}^{11}+{\widetilde{u_{a} 2}}^{2}+\widetilde{u_{a 2}}{ }^{33}\right)+ \\
\left.\delta_{3}\left(\widetilde{u_{a 3}}{ }^{11}+{\widetilde{u_{a 3}}}^{2}+{\widetilde{u_{a 3}}}^{33}\right)\right\}=0 .
\end{gathered}
$$

Now we form the vorticity equation, which can then be combined with the mass continuity equation to eliminate the ageostrophic velocity. Take $\widetilde{(.)}^{2}$ applied to $(52)$ minus $\widetilde{(.)}$ applied to (51) to obtain

$$
\begin{aligned}
& \partial_{t}\left({\widetilde{u_{3}}}^{2}-{\widetilde{u_{2}}}^{3}\right)-\frac{f_{0}}{\sqrt{3}}\left({\widetilde{u_{a 1}}}^{2} 2-{\widetilde{u_{a 2}}}^{12}-{\widetilde{u_{a 3}}}^{13}+{\widetilde{u_{a 1}}}^{33}\right) \\
& -\frac{1}{\sqrt{3}}\left({\widetilde{f^{\prime}{\widetilde{u_{1}}}^{2}}}^{2}-{\widetilde{f^{\prime}{\widetilde{u_{2}}}^{1}}}^{2}-{\widetilde{f^{\prime}{\widetilde{u_{3}}}^{3}}}^{3}+{\widetilde{f^{\prime}{\widetilde{u_{1}}}^{3}}}^{3}\right)-\frac{d^{2}}{6 \sqrt{3}}\left({\widetilde{B_{3}}}^{2}-{\widetilde{B_{2}}}^{3}\right)=0 \text {. }
\end{aligned}
$$

Substitute for the first term using (37), use (57) to simplify the terms in $f^{\prime}$, and use (38) and (39) to replace the resulting terms $T_{2}\left({\widetilde{u_{1}}}^{2}-{\widetilde{u_{2}}}^{1}\right)$ and $T_{3}\left({\widetilde{u_{3}}}^{1}-{\widetilde{u_{1}}}^{3}\right)$, to obtain 


$$
\begin{aligned}
& \partial_{t} \delta_{1} \Phi+ \frac{f_{0}^{2}}{3}\left({\widetilde{u_{a 1}}}^{22}-{\widetilde{u_{a 2}}}^{12}-{\widetilde{u_{a 3}}}^{13}+{\widetilde{u_{a 1}}}^{33}\right) \\
&+ \frac{f_{0} f^{\prime}}{3}\left({\widetilde{u_{1}}}^{22}-{\widetilde{u_{2}}}^{12}-{\widetilde{u_{3}}}^{13}+{\widetilde{u_{1}}}^{33}\right) \\
&+\frac{d^{2}}{6 \sqrt{3}}\left\{T_{2}\left(\delta_{3} \Phi\right)-T_{3}\left(\delta_{2} \Phi\right)\right\}=0 . \\
&+\frac{d^{2} f_{0}}{18}\left({\widetilde{B_{3}}}^{2}-{\widetilde{B_{2}}}^{3}\right)
\end{aligned}
$$

In an analogous way, or by permutation of indices, we also have

$$
\begin{aligned}
& \partial_{t} \delta_{2} \Phi+ \frac{f_{0}^{2}}{3}\left({\widetilde{u_{a 2}}}^{33}-{\widetilde{u_{a 3}}}^{23}-{\widetilde{u_{a 1}}}^{21}+{\widetilde{u_{a 2}}}^{11}\right) \\
&+ \frac{f_{0} f^{\prime}}{3}\left({\widetilde{u_{2}}}^{33}-{\widetilde{u_{3}}}^{23}-{\widetilde{u_{1}}}^{21}+{\widetilde{u_{2}}}^{11}\right) \\
&+\frac{d^{2}}{6 \sqrt{3}}\left\{T_{3}\left(\delta_{1} \Phi\right)-T_{1}\left(\delta_{3} \Phi\right)\right\}=0 \\
&+\frac{d^{2} f_{0}}{18}\left({\widetilde{B_{1}}}^{3}-{\widetilde{B_{3}}}^{1}\right)=0
\end{aligned}
$$

and

$$
\begin{aligned}
\partial_{t} \delta_{3} \Phi+ & \frac{f_{0}^{2}}{3}\left({\widetilde{u_{a 3}}}^{11}-{\widetilde{u_{a 1}}}^{31}-{\widetilde{u_{a 2}}}^{32}+{\widetilde{u_{a 3}}}^{22}\right) \\
+ & \frac{f_{0} f^{\prime}}{3}\left({\widetilde{u_{3}}}^{11}-{\widetilde{u_{1}}}^{31}-{\widetilde{u_{2}}}^{32}+{\widetilde{u_{3}}}^{22}\right) \\
+\frac{d^{2}}{6 \sqrt{3}}\left\{T_{1}\left(\delta_{2} \Phi\right)-T_{2}\left(\delta_{1} \Phi\right)\right\} & =0 .
\end{aligned}
$$

Now take $\delta_{1}(69)+\delta_{2}(70)+\delta_{3}(71)$ to form the vorticity equation. Use the identity (30) to rewrite the ageostrophic velocity terms, by analogy with the step from (65) to (66), and hence eliminate them using the mass continuity equation (67). Using the fact that the operators $\delta_{j}$ commute with the operators $T_{k}, j, k=1,2,3$, we find that all terms involving $T_{k}$ cancel, leaving

$$
\begin{array}{r}
\partial_{t}\left\{\left(\delta_{1} \delta_{1} \Phi+\delta_{2} \delta_{2} \Phi+\right.\right. \\
\left.\left.+\delta_{3} \delta_{3} \Phi\right)-\frac{f_{0}^{2}}{2 \Phi_{0}}\left(\widetilde{\Phi}^{11}+\widetilde{\Phi}^{22}+\widetilde{\Phi}^{33}\right)\right\} \\
+\frac{f_{0}}{3}\left\{\delta_{1}\left[f^{\prime}\left({\widetilde{u_{1}}}^{22}-{\widetilde{u_{2}}}^{12}-{\widetilde{u_{3}}}^{13}+{\widetilde{u_{1}}}^{33}\right)\right]\right. \\
+\delta_{2}\left[f^{\prime}\left({\widetilde{u_{2}}}^{33}-{\widetilde{u_{3}}}^{23}-{\widetilde{u_{1}}}^{21}+{\widetilde{u_{2}}}^{11}\right)\right]
\end{array}
$$




$$
\begin{gathered}
\left.+\delta_{3}\left[f^{\prime}\left({\widetilde{u_{3}}}^{11}-{\widetilde{u_{1}}}^{31}-{\widetilde{u_{2}}}^{32}+{\widetilde{u_{3}}}^{2}\right)\right]\right\} \\
+\frac{d^{2} f_{0}}{18}\left\{\delta_{1}\left({\widetilde{B_{3}}}^{2}-{\widetilde{B_{2}}}^{3}\right)+\delta_{2}\left({\widetilde{B_{1}}}^{3}-{\widetilde{B_{3}}}^{1}\right)+\delta_{3}\left({\widetilde{B_{2}}}^{1}-{\widetilde{B_{1}}}^{2}\right)\right\}=0 .
\end{gathered}
$$

Now use the one dimensional identitity

$$
\Delta(a b)=(\Delta a) \bar{b}+\bar{a}(\Delta b)
$$

to re-express the terms involving $f^{\prime}$, then use (65) to eliminate some of the resulting terms and (37)-(39) to simplify the rest, to obtain

$$
\begin{aligned}
& \partial_{t}\left\{\left(\delta_{1} \delta_{1} \Phi+\delta_{2} \delta_{2} \Phi+\delta_{3} \delta_{3} \Phi\right)-\frac{f_{0}^{2}}{2 \Phi_{0}}\left(\widetilde{\Phi}^{11}+\widetilde{\Phi}^{22}+\widetilde{\Phi}^{33}\right)\right\} \\
& +\frac{1}{\sqrt{3}}\left\{\beta_{1}{\overline{\left(\delta_{3} \widetilde{\Phi}^{2}-\delta_{2} \widetilde{\Phi}^{3}\right)}}^{1}+\beta_{2}{\overline{\left(\delta_{1} \widetilde{\Phi}^{3}-\delta_{3} \widetilde{\Phi}^{1}\right)}}^{2}+\beta_{3}{\overline{\left(\delta_{2} \widetilde{\Phi}^{1}-\delta_{1} \widetilde{\Phi}^{2}\right)}}^{3}\right\} \\
& +\frac{d^{2} f_{0}}{18}\left\{\delta_{1}\left({\widetilde{B_{3}}}^{2}-{\widetilde{B_{2}}}^{3}\right)+\delta_{2}\left({\widetilde{B_{1}}}^{3}-{\widetilde{B_{3}}}^{1}\right)+\delta_{3}\left({\widetilde{B_{2}}}^{1}-{\widetilde{B_{1}}}^{2}\right)\right\} \\
& =0 \text {. }
\end{aligned}
$$

We now have two equations, the solvability condition (60) and the vorticity equation (74), that have constant coefficients. We can therefore seek solutions proportional to $\exp \{\mathrm{i}(\mathbf{k} \cdot \mathbf{x}-\omega t)\}$, and use the fact that the solution is of the form (41); for a given wave vector $\mathbf{k}$ the two equations determine the two unknowns $\mu / \Phi$, which ties down the wave structure, and $\omega$, the wave frequency. Substituting (41) into (60) and simplifying leads to

$$
\omega(A \mu-R \Phi)+S \Phi+M \mu-N \Phi=0,
$$

while substituting (41) into (74) and simplifying leads to

$$
\omega W \Phi-V \Phi+P \Phi-Q \mu=0,
$$

where

$$
\begin{aligned}
& A=\frac{1}{d \sqrt{3}}\left(a_{1}^{2}+a_{2}^{2}+a_{3}^{3}\right), \\
& R=\frac{1}{d \sqrt{3}}\left(a_{1} p_{1}+a_{2} p_{2}+a_{3} p_{3}\right), \\
& S=\frac{1}{3}\left(s_{1} t_{1}+s_{2} t_{2}+s_{3} t_{3}\right),
\end{aligned}
$$




$$
\begin{aligned}
& M=\frac{1}{9}\left\{\left(a_{3} b_{31}-a_{2} b_{21}\right) a_{1}+\left(a_{1} b_{12}-a_{3} b_{32}\right) a_{2}+\left(a_{2} b_{23}-a_{1} b_{13}\right) a_{3}\right\}, \\
& N=\frac{1}{9}\left\{\left(a_{3} b_{31}-a_{2} b_{21}\right) p_{1}+\left(a_{1} b_{12}-a_{3} b_{32}\right) p_{2}+\left(a_{2} b_{23}-a_{1} b_{13}\right) p_{3}\right\}, \\
& W=\frac{4}{d^{2}}\left(s_{1}^{2}+s_{2}^{2}+s_{3}^{2}\right)+\frac{f_{0}^{2}}{2 \Phi_{0}}\left(a_{1}^{2}+a_{2}^{2}+a_{3}^{2}\right), \\
& V=\frac{2}{d \sqrt{3}}\left(\beta_{1} c_{1} q_{1}+\beta_{2} c_{2} q_{2}+\beta_{3} c_{3} q_{3}\right) \\
& P=\frac{4}{9 \sqrt{3} d}\left\{\left(q_{3} b_{31}-q_{2} b_{21}\right) p_{1}+\left(q_{1} b_{12}-q_{3} b_{32}\right) p_{2}\right. \\
& \left.+\left(q_{2} b_{23}-q_{1} b_{13}\right) p_{3}\right\} \\
& Q=\frac{4}{9 \sqrt{3} d}\left\{\left(q_{3} b_{31}-q_{2} b_{21}\right) a_{1}+\left(q_{1} b_{12}-q_{3} b_{32}\right) a_{2}\right. \\
& \left.+\left(q_{2} b_{23}-q_{1} b_{13}\right) a_{3}\right\}, \\
& b_{12}=\beta_{1} s_{1} c_{2}+\beta_{3} s_{3} / 4, \\
& t_{1}=\beta_{3} s_{3} c_{2}+\beta_{2} s_{2} c_{3}+\beta_{1} s_{1} / 2=b_{32}+b_{23}, \\
& q_{1}=s_{2} a_{3}-s_{3} a_{2},
\end{aligned}
$$

with other $b_{j k}, t_{j}$ and $q_{j}$ defined by cyclic permutation of indices.

Eliminating $\mu / \Phi$ gives the dispersion relation, a quadratic in $\omega$ :

$$
\begin{array}{r}
\omega^{2} A W+\omega\{A(P-V)-R Q+M W\}+M(P-V)+Q(S-N) \\
=0 .
\end{array}
$$

The two roots for $\omega$ correspond to the two Rossby mode branches. It may be verified that the coefficient of $\omega^{2}$ is independent of $\boldsymbol{\beta}$, the coefficient of $\omega$ is proportional to $|\boldsymbol{\beta}|$, and the constant term is proportional to $|\boldsymbol{\beta}|^{2}$, implying that both roots for $\omega$ are proportional to $|\boldsymbol{\beta}|$, as one would hope for Rossby modes. Note also that, because the spatial discretization is energy conserving, the roots for $\omega$ must be real. 


\subsection{Scheme (ii)}

The derivation of the dispersion relation for scheme (ii) follows the same steps and is very similar in detail to that for scheme (i). The only differences arise in the terms involving $f^{\prime}$ and the way they are simplified. The dispersion relation is found to take the same form as (89) provided we redefine four of the coefficients:

$$
\begin{aligned}
& M=0 \\
& N=\frac{1}{18}\left\{\left(a_{3} t_{2}-a_{2} t_{3}\right) p_{1}+\left(a_{1} t_{3}-a_{3} t_{1}\right) p_{2}+\left(a_{2} t_{1}-a_{1} t_{2}\right) p_{3}\right\} \\
& P=\frac{2}{9 \sqrt{3} d}\left\{\left(q_{3} t_{2}-q_{2} t_{3}\right) p_{1}+\left(q_{1} t_{3}-q_{3} t_{1}\right) p_{2}+\left(q_{2} t_{1}-q_{1} t_{2}\right) p_{3}\right\} \\
& Q=\frac{2}{9 \sqrt{3} d}\left\{\left(q_{3} t_{2}-q_{2} t_{3}\right) a_{1}+\left(q_{1} t_{3}-q_{3} t_{1}\right) a_{2}+\left(q_{2} t_{1}-q_{1} t_{2}\right) a_{3}\right\}
\end{aligned}
$$

\subsection{Scheme (iii)}

The derivation of the dispersion relation for scheme (iii) is again very similar in detail to that for scheme (i). The dispersion relation is found to take the same form as (89) provided we redefine the four coefficients $M, N, P$ and $Q$ by replacing $b_{i j}$ by $b_{j i}$ throughout (80), (81), (84) and (85).

\subsection{Results}

It is useful first to check the behaviour of the numerical dispersion relation in the well-resolved limit $|\mathbf{k}| d \ll 1$. By examining how the different contributions scale for small $|\mathbf{k}| d$, the dominant contributions to the coefficients in (89) can be identified; (89) becomes

$$
W \omega^{2}-V \omega+C \approx 0
$$

where $C=[-M V+Q(S-N)] / A$ is $O\left((|\mathbf{k}| d)^{4}\right)$. One of the roots, the primary Rossby mode branch, is relatively large and is the same for all three schemes:

$$
\omega \approx \frac{V}{W} \approx \frac{(\alpha l-\beta k) \Phi_{0}}{f_{0}^{2}+\Phi_{0}|\mathbf{k}|^{2}}
$$


The frequency clearly agrees with the continuous case in this well-resolved limit; this is also evident from the central regions of the panels in Figs. 4, 5, and 8 below. The other root, the secondary Rossby mode branch,

$$
\omega \approx \frac{C}{V}
$$

is relatively small, scaling like $O\left(|\mathbf{k}|^{3} d^{4}\right)$; its mathematical form is rather complicated and is different for each of the three schemes.

For arbitrary $|\mathbf{k}| d$, motivated by the fact that the Rossby mode frequency for the continuous equations goes to zero for wave vectors satisfying $\alpha l-\beta k=$ 0 , we identify the primary branch of the numerical Rossby mode dispersion relation as given by the "+" root in the formula

$$
\omega=\frac{-b \pm \operatorname{sign}(\alpha l-\beta k)\left(b^{2}-4 a c\right)^{1 / 2}}{2 a}
$$

and the secondary branch as given by the "-" root, where $a=A W, b=$ $A(P-V)-R Q+M W$, and $c=M(P-V)+Q(S-N)$. The results below justify this choice, confirming that the primary branch is indeed almost always closer than the secondary branch to the continuous equation Rossby mode frequency for that wave vector.

Figure 4 shows the quasigeostrophic Rossby mode dispersion relations for the continuous equations and for the primary Rossby mode branch of the three numerical schemes analysed above for a case with $\alpha=0$. All three schemes are very accurate for small wavenumbers and show some artificial slowing for larger wavenumbers. Scheme (i) is noticeably more accurate than the other two, and scheme (ii) shows some anomalous behaviour for the very largest resolvable wavenumbers.

Figure 5 shows analogous results when the coordinate system and grid are rotated through $90^{\circ}$. At first glance the results are qualitatively very similar to those in Fig. 4. Again, scheme (i) is noticeably more accurate than the other two and scheme (ii) shows some anomalous behaviour for the largest resolvable wavenumbers. Similar results are also found for other rotation angles (not shown). The fact that these numerical dispersion relations are almost insensitive to grid orientation is an improvement over the corresponding behaviour on a quadrilateral C-grid. In particular, for a quadrilateral C-grid aligned with the north-south and east-west directions, short east-west wavelengths are significantly retarded, with frequency going to zero for the shortest resolvable east-west scales [23]. This is not the case for any of the hexagonal C-grid schemes examined here; Rossby mode frequencies go to zero only when the east-west component of the wave vector goes to zero, in agreement with the 

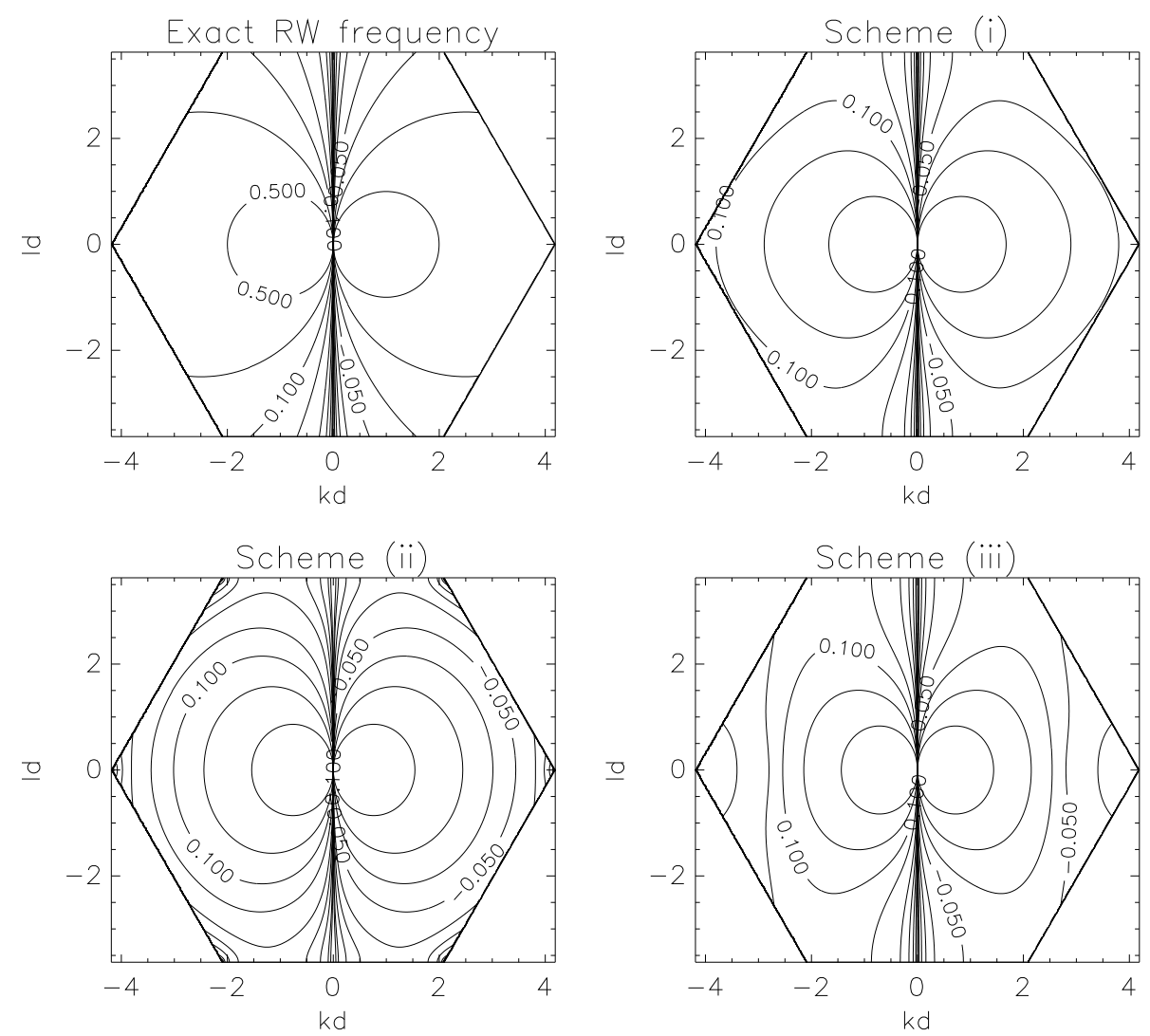

Fig. 4. Values of $\omega \times 10^{6}$ versus $k d$ and $l d$ for quasigeostrophic Rossby mode dispersion relations. Top left: continuous equations. Top right: scheme (i) primary branch. Bottom left: scheme (ii) primary branch. Bottom right: scheme (iii) primary branch. Contour values are 1, 2, and 5 times powers of 10 . The parameters used are $d=10^{5} \mathrm{~m}, \Phi_{0}=10^{5} \mathrm{~m}^{2} \mathrm{~s}^{-2}, f_{0}=10^{-4} \mathrm{~s}^{-1}, \alpha=0 \mathrm{~s}^{-1} \mathrm{~m}^{-1}$, and $\beta=10^{-11} \mathrm{~s}^{-1} \mathrm{~m}^{-1}$, implying a resolution factor $d / \lambda=0.0316$.

continuous dispersion relation. Thus, the hexagonal C-grid has better isotropy properties and, for short east-west wavelengths on the primary Rossby mode branch, better accuracy, than a quadrilateral C-grid.

An interesting feature of Fig. 5, however, is the fact that for all three schemes the primary branch numerical Rossby mode frequency changes discontinuously at $l d=0$. More generally, a similar discontinuity occurs for all non-zero values of $\alpha$ along the line $\alpha l-\beta k=0$ in wave vector space. From (97), it is clear that the discontinuity occurs as the root of the quadratic corresponding to the primary branch of the dispersion relation switches. Figure 6 shows several onedimensional sections through the numerical dispersion relation for scheme (i) for the same case shown in Fig. 5. The discontinuity is almost unnoticeable for smaller values of $k d$, but it becomes increasingly noticeable, and the numerical dispersion relation becomes less accurate, for larger $k d$. Similar results are found for other grid orientations (not shown). This figure also makes it clear that the definition of the primary and secondary branches used here (97) is 

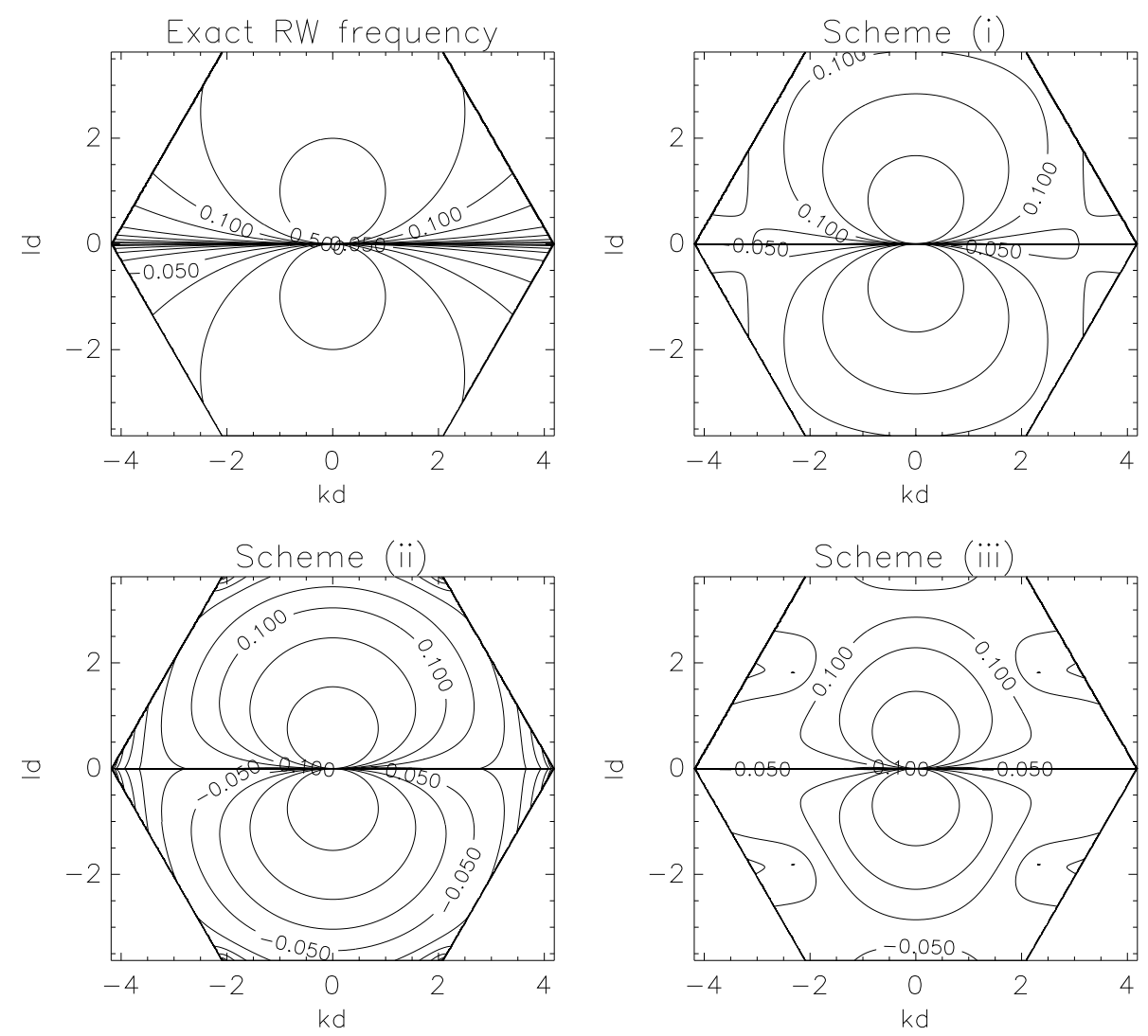

Fig. 5. As in Fig. 4, except that $\alpha=10^{-11} \mathrm{~s}^{-1} \mathrm{~m}^{-1}$ and $\beta=0 \mathrm{~s}^{-1} \mathrm{~m}^{-1}$.

reasonable.

Figure 7 shows the dispersion relations for the secondary Rossby mode branch of the three numerical schemes. All three schemes correctly give zero frequency for $k d=0$. However, they all appear to give unrealistic eastward propagation, with $\omega<0$ for $k d<0$ and $\omega>0$ for $k d>0$. Typical magnitudes of the frequencies are much smaller than for the primary branch. There are striking differences between the three schemes, with the scheme (ii) and scheme (iii) dispersion relations showing quite complex structure. For all three schemes, the secondary branch dispersion relations are much more sensitive to grid orientation than the primary branches (not shown). The secondary Rossby mode branch is discussed in more detail in section 6

Figure 8 is like Fig. 4 but for a case with poorly resolved Rossby radius. Although the C-grid is not expected to perform well in this parameter regime, schemes (ii) and (iii) remain accurate for the small wavenumber part of the spectrum and scheme (i) remains accurate for almost all of the spectrum. 

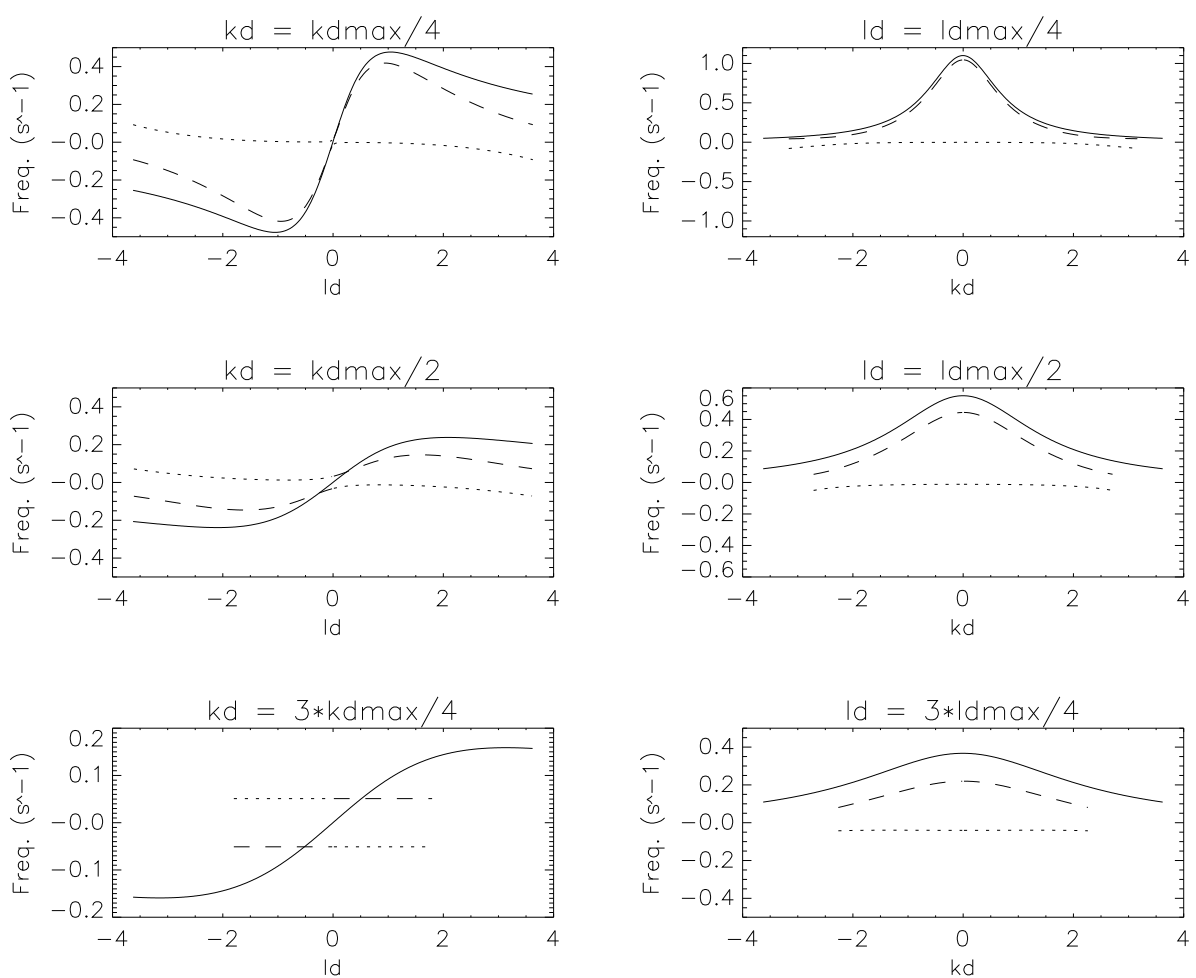

Fig. 6. Quasigeostrophic Rossby mode dispersion relations. Solid curves: continuous equations. Dashed curves: scheme (i) primary branch. Dotted curves: scheme (i) secondary branch. Left hand panels, top to bottom: frequency versus $l d$ at $k d=1 / 4,1 / 2$ and $3 / 4$ times $k d_{\max }=4 \pi / 3$ respectively. Right hand panels, top to bottom: frequency versus $k d$ at $l d=1 / 4,1 / 2$ and $3 / 4$ times $l d_{\max }=2 \pi / \sqrt{3}$ respectively. Note the different frequency scales on the different panels. Parameters are the same as in Fig. 5.

\section{Comparison with direct numerical calculation of normal modes}

As a valuable check on the results of sections 2 and 4 , the normal mode frequencies for schemes (i)-(iii) can also be calculated directly via numerical implementations of the schemes. Consider the linearized shallow water equations in a Cartesian $\beta$-plane channel of width $D$ : the channel extends infinitely in the east-west direction with impermiable walls at the northern and southern boundaries, and $f=f_{0}+\beta y$ where $y$ is the northward coordinate.

Because of the symmetry in the $x$-direction, this system has normal modes proportional to $\exp (\mathrm{i} k x)$. By making the $f$-plane approximation the approximate frequencies for the inertio-gravity modes of the continuous system can be found. For the parameters used below this is an excellent approximation. Alternatively, by making the quasigeostrophic approximation the approximate frequencies for the Rossby modes of the continuous system can be found. Again, for the parameters used below this is an excellent approximation. 

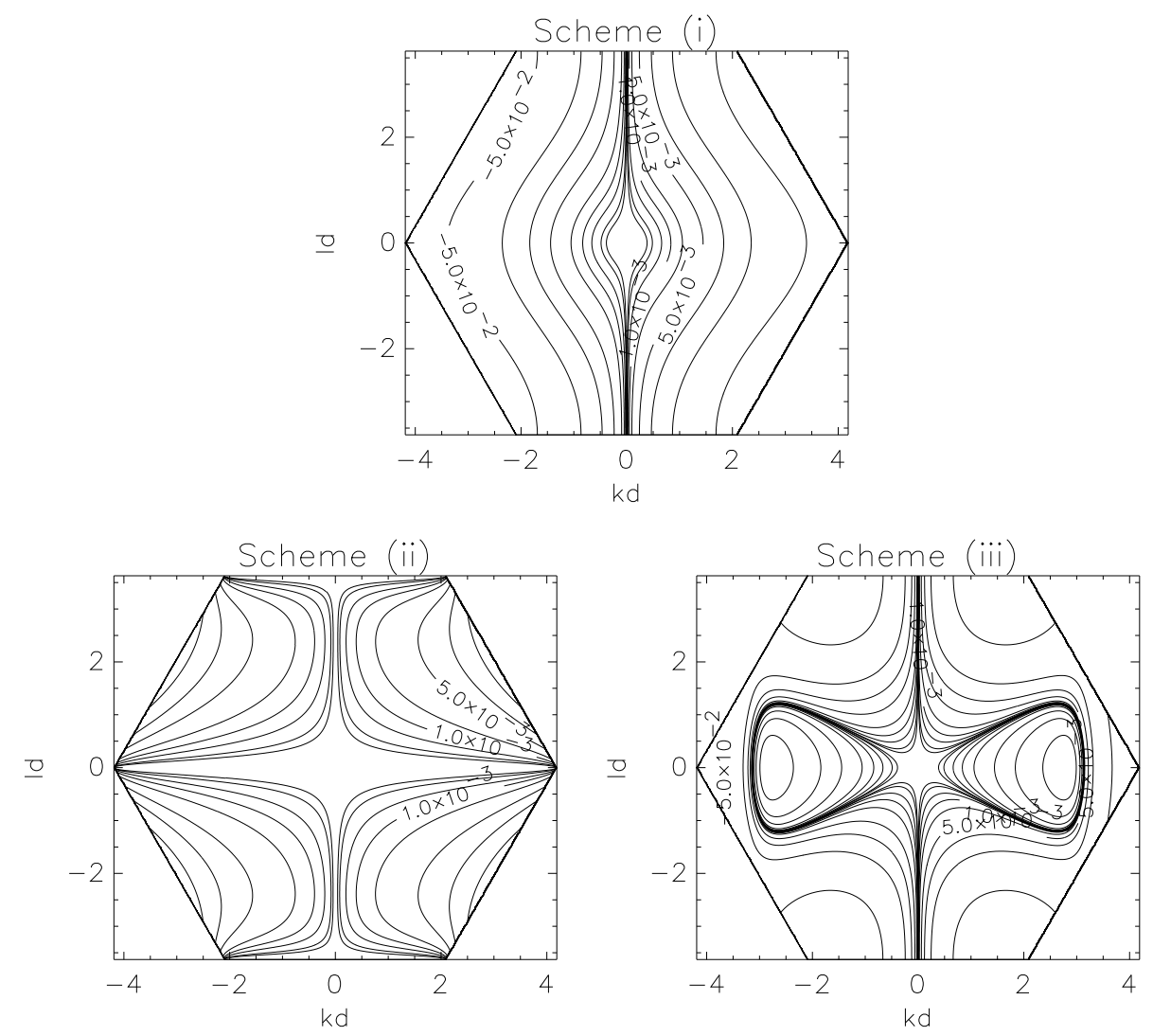

Fig. 7. Values of $\omega \times 10^{6}$ versus $k d$ and $l d$ for secondary branch of the numerical quasigeostrophic Rossby mode dispersion relations. Top: scheme (i). Bottom left: scheme (ii). Bottom right: scheme (iii). The parameters are the same as in Fig. 4.

We can also discretize this system using a hexagonal C-grid. Rows of hexagons are aligned parallel to the channel, and the boundary condition $v=0$ at the northern and southern boundaries is approximated by setting the components $u_{2}$ and $u_{3}$ to zero there. The normal mode frequencies of the discrete system can be found by expressing the discrete equations in matrix form

$$
-\mathrm{i} \omega \mathbf{s}=\mathbf{M} \mathbf{s}
$$

where $\mathbf{s}$ is the state vector comprising a list of all the $u_{1}, u_{2}, u_{3}$ and $\Phi$ values, and then finding the eigenvalues of $\mathbf{M}$. The symmetry of the problem again implies that normal modes will be proportional to $\exp (\mathrm{i} k x)$. Specifying $k$ and taking out the exponential factor allows the problem to be reduced from two dimensions to one (though keeping all the properties of the two dimensional scheme) and greatly reducing the size of $\mathbf{M}$. The matrix $\mathbf{M}$ can be constructed numerically by repeated calls to a subroutine that calculates the tendencies of $u_{1}, u_{2}, u_{3}$ and $\Phi$ given their gridded values. This procedure gives the normal mode frequencies for the discrete, but otherwise unapproximated, channel problem. 

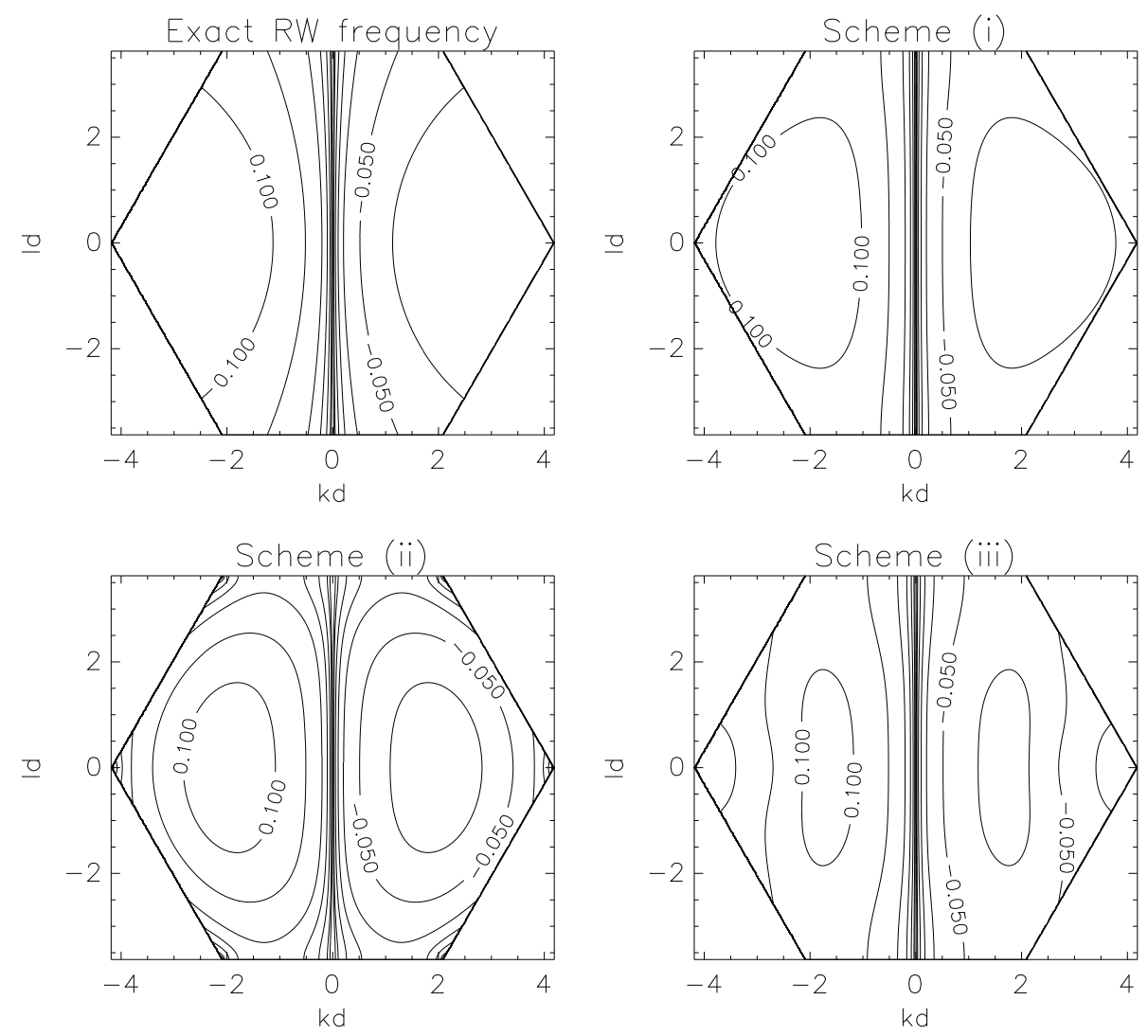

Fig. 8. As in Fig. 4, except that $\Phi_{0}=10 \mathrm{~m}^{2} \mathrm{~s}^{-2}$, implying $d / \lambda=3.16$.

Finally, for comparison, approximate normal mode frequencies for the discrete problem are given by finding the quantized values of the meridional component $l$ of the wave vector allowed by the boundary conditions and substituting those, along with the specified $k$, into the $f$-plane discrete inertio-gravity mode dispersion relation (the non-zero roots of (32)) and the quasigeostrophic $\beta$ plane Rossby mode dispersion relation (89).

Figure 9 shows the results of these three calculations plotted together for scheme (i). The inertio-gravity mode frequencies for the numerical scheme are very accurate for small wavenumbers but are somewhat slowed for large wavenumbers, in agreement with Fig. 2. (The inertio-gravity mode frequencies in Fig. 9 correspond to a section $k d=\pi / 10, l d=(0,2 \pi / \sqrt{3})$ in the top left and bottom left panels of Fig. 2.) There is almost exact symmetry between eastward and westward inertio-gravity modes.

The primary $(\omega<0)$ Rossby mode branch for the numerical scheme is also very accurate across the whole spectrum. (This branch corresponds to a section $k d=\pi / 10, l d=(0,2 \pi / \sqrt{3})$ in the top left and bottom left panels of Fig. 4.) Interestingly, the largest relative errors occur around the middle of the spectrum. 

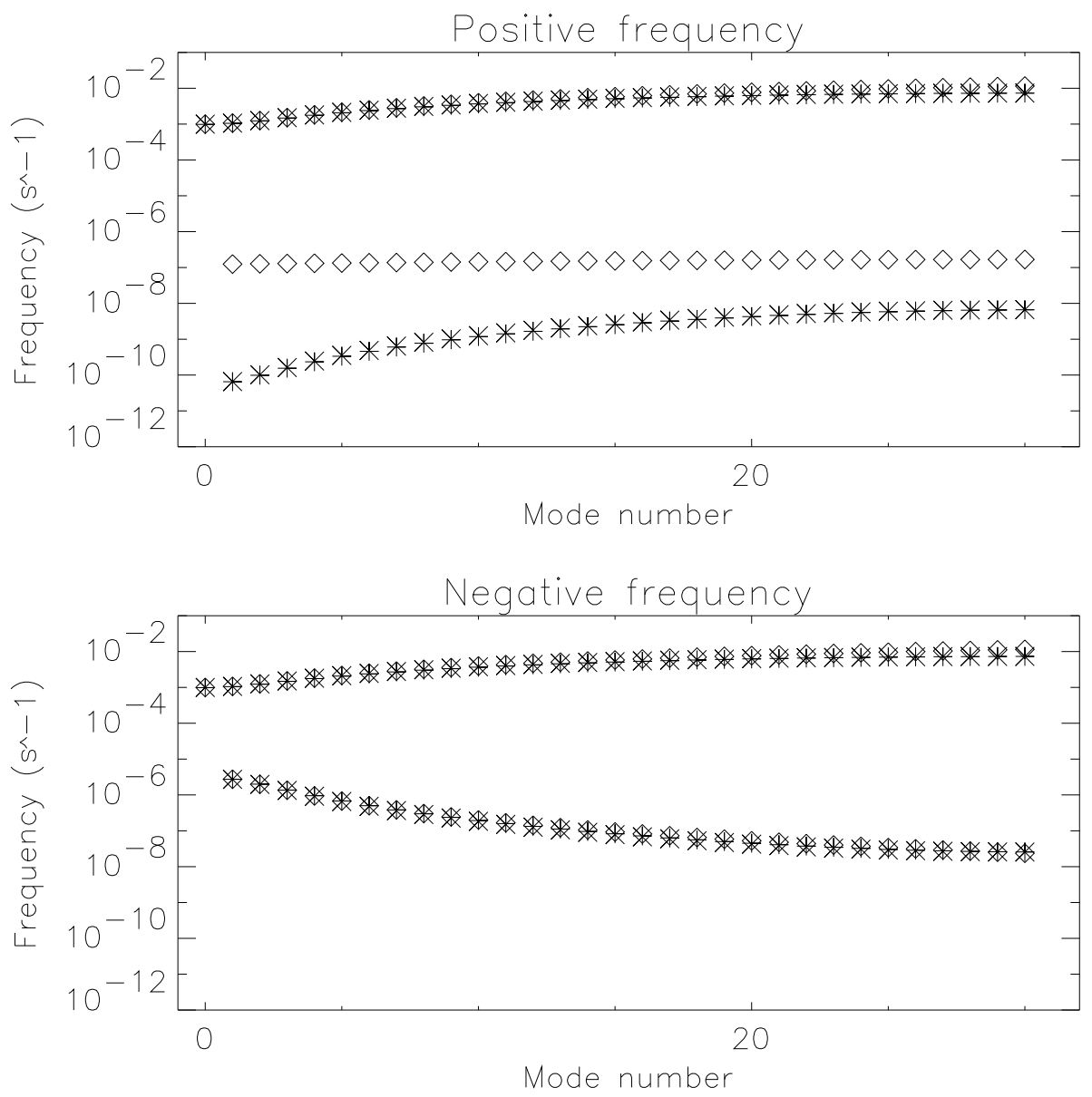

Fig. 9. Comparison of continuous and numerical dispersion relations for a $\beta$-plane channel, showing frequency versus meridional mode number, defined as the number of zeros in the $\Phi$ field in a section across the channel. Diamonds correspond to the continuous governing equations. Frequencies greater than $10^{-4} \mathrm{~s}^{-1}$ in magnitude are inertio-gravity modes, obtained using the $f$-plane approximation. Frequencies smaller than $10^{-4} \mathrm{~s}^{-1}$ in magnitude are Rossby modes, obtained using the quasigeostrophic $\beta$-plane approximation; the positive frequency Rossby mode branch is computed using aliased wave vectors - see section 6 . Crosses $(\times)$ correspond to theoretical frequencies for scheme (i), given by the non-zero roots of (32) for inertio-gravity modes and by (89) for Rossby modes. Plus (+) symbols are normal mode frequencies for scheme (i) computed directly via the eigenvalue problem (98). Note that plus and cross symbols overlay each other almost exactly. The parameters used are $d=10^{5} \mathrm{~m}, \Phi_{0}=10^{5} \mathrm{~m}^{2} \mathrm{~s}^{-2}, f_{0}=10^{-4} \mathrm{~s}^{-1}, \alpha=0 \mathrm{~s}^{-1} \mathrm{~m}^{-1}, \beta=10^{-11} \mathrm{~s}^{-1} \mathrm{~m}^{-1}$, and $k d=\pi / 10$. For the direct normal mode calculation $N=30$ rows of hexagons were used giving a channel width $30 d \sqrt{3} / 2 \approx 2.6 \times 10^{6} \mathrm{~m}$.

The secondary Rossby mode branch for the numerical scheme has $\omega>0$, but with $|\omega|$ significantly smaller than for the primary branch and with largest $|\omega|$ for the largest mode numbers. (This branch corresponds to a section $k d=$ $\pi / 10, l d=(0,2 \pi / \sqrt{3})$ in the top panel of Fig. 7 .) The secondary Rossby mode branch will be discussed further in section 6 . 
For the inertio-gravity modes, the excellent agreement between the theoretical frequencies for the numerical scheme and those calculated directly using (98) provides a valuable check on the correctness of the calculations as well as the accuracy of the $f$-plane approximation. For Rossby modes there is also excellent agreement, but this is somewhat fortuitous. The theoretical frequencies are calculated for a single Fourier mode of wave vector $(k, l)$ in an infinite or periodic domain, whereas the calculation (98) is for a channel. In order for the two calculations to agree, the $(k, l)$ Fourier mode must have the same frequency as the $(k,-l)$ mode, which it does, and the velocity structures of the $(k, l)$ and $(k,-l)$ modes must allow the channel boundary condition to be satisfied by a suitable superposition; however, a detailed examination of the velocity structures shows that this is not the case for the Rossby modes, though it is almost true for many modes. The effects are not noticeable for scheme (i), but they are noticeable for scheme (ii) and are significant for scheme (iii) (see below).

Figure 10 is similar to Fig. 9 but for scheme (ii). Again the inertio-gravity modes are well captured by the scheme, though with some slowing at large wavenumbers. The primary Rossby mode branch is quite accurately captured, though there is significant slowing for large wavenumbers making it less accurate than for scheme (i). As for scheme (i), the secondary Rossby mode branch has $\omega>0$ with $|\omega|$ much smaller than for the primary branch; however, for scheme (ii) the frequency does not vary monotonically with mode number. For large mode numbers there is a small but noticeable discrepancy between the theoretical numerical Rossby mode frequencies and those calculated using (98) due to the channel boundary condition.

Figure 11 is similar to Fig. 9 but for scheme (iii). Once again the inertiogravity modes are well captured by the scheme, though with some slowing at large wavenumbers. The primary Rossby mode branch is more accurate than for scheme (ii) but less accurate than for scheme (i). The secondary Rossby mode branch again has $\omega>0$, with $|\omega|$ much smaller than for the primary branch modes. The effects of the channel boundary condition are much greater for scheme (iii): for the secondary branch modes labelled with smaller mode numbers there is now a significant discrepancy between the theoretical numerical frequencies and those calculated using (98); also, three negative frequency modes lie well away from the main dispersion curve.

\section{The secondary Rossby mode branch}

A key question is whether or not the modes in the secondary Rossby mode branch are useful approximations to modes of the continuous equations, that is, whether or not the extra degrees of freedom in the wind field translate, in 

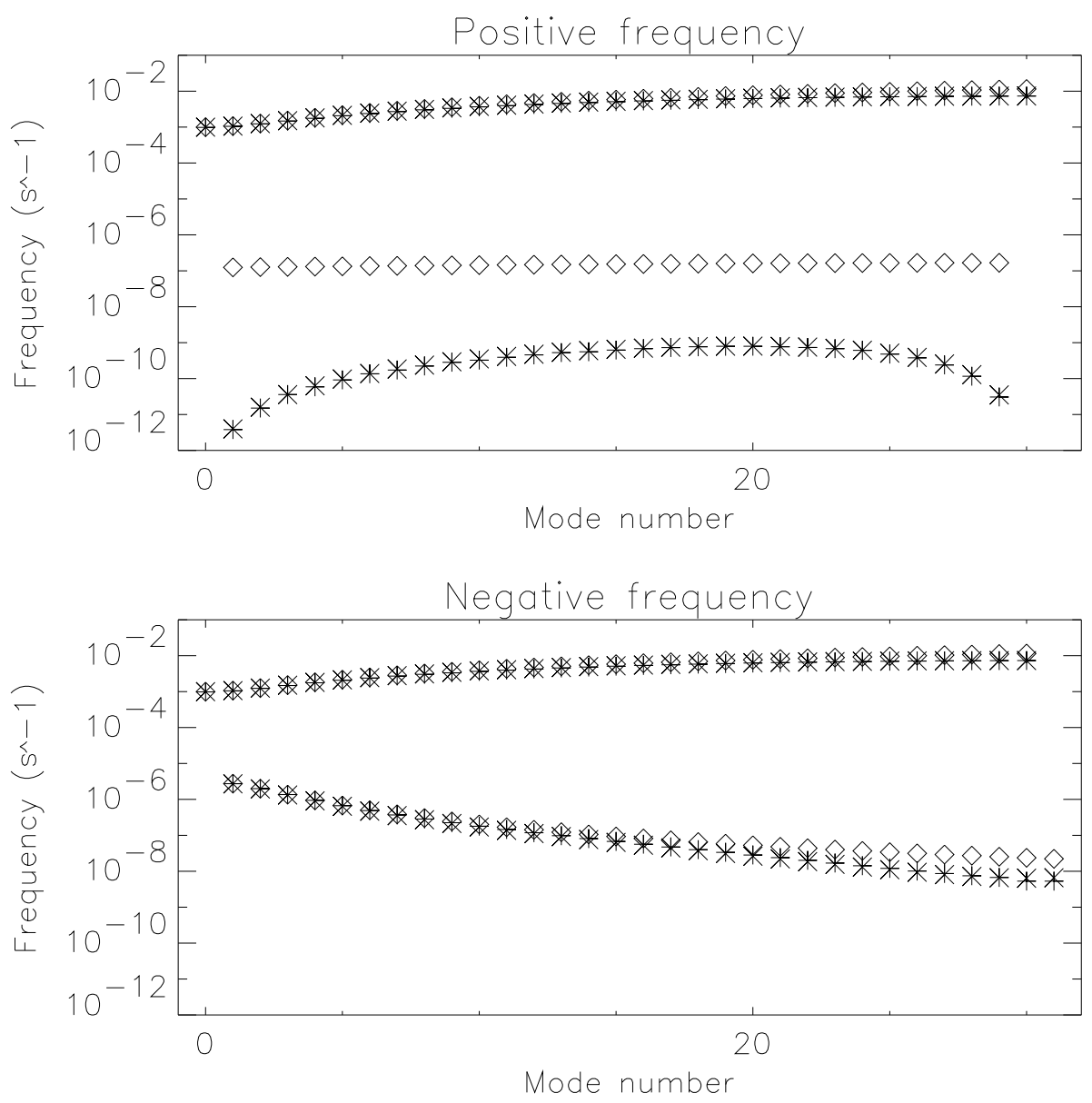

Fig. 10. As in Fig. 9 but for scheme (ii).

effect, into extra resolution.

For a single field proportional to $\exp (\mathbf{i k . x})$ defined at $\Phi$ points (say) the resolvable wave vectors all lie within the hexagonal regions indicated in Figs. 2-5 and 7-8. Any field with a wave vector outside this region is aliased into the hexagonal region. Specifically, a mode of wave vector $\mathbf{k}$ is indistinguishable, on the grid, from one of wave vector $\mathbf{k}+\mathbf{k}^{\prime}$ where

$$
\mathbf{k}^{\prime} d=n_{1}(0,4 \pi / \sqrt{3})+n_{2}(-2 \pi,-2 \pi / \sqrt{3})+n_{3}(2 \pi,-2 \pi / \sqrt{3})
$$

for any integers $n_{1}, n_{2}, n_{3}$.

Now consider fields with three components $(u, v, \Phi)$ required to describe shallow water flow. For any given $\mathbf{k}$, the space of such fields is spanned by the eastward gravity, westward gravity, and Rossby normal modes of the continuous equations for that $\mathbf{k}$. For $\mathbf{k}$ within the hexagonal region of resolvable wave vectors, the eastward gravity, westward gravity, and primary branch Rossby normal modes of the discrete equations are useful approximations to these continuous normal modes. Therefore, if the secondary Rossby mode branch is an approximation to anything physical, it must be to something with a $\mathbf{k}$ 

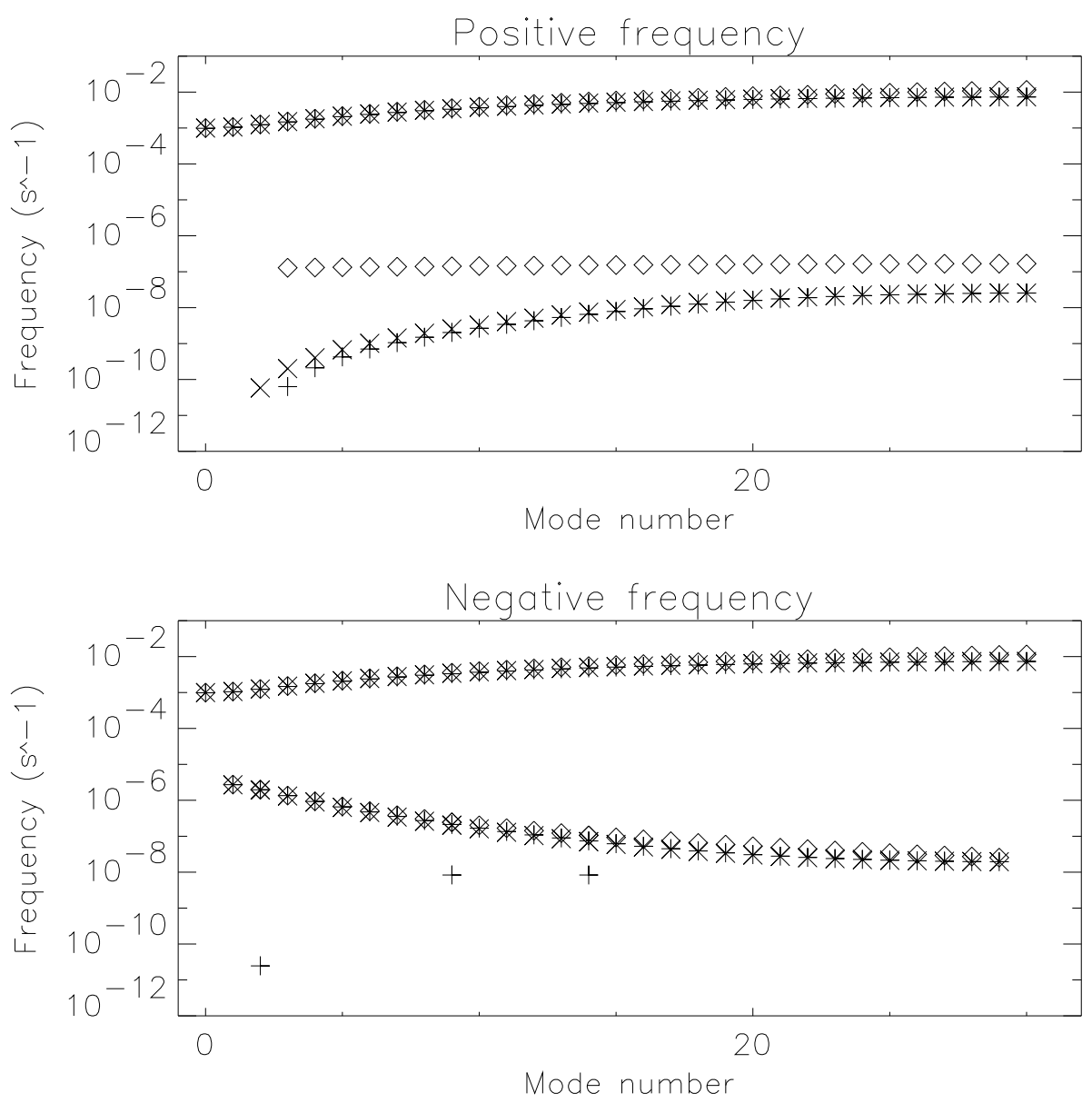

Fig. 11. As in Fig. 9 but for scheme (iii).

outside the hexagonal region.

This can be illustrated by considering the Rossby modes on the two branches in the limit $|\mathbf{k}| \rightarrow 0$. The primary branch mode has $\mu / \Phi \rightarrow 0$, and, since the $p_{j}$ also tend to zero, by (41) the velocity field tends to zero. Thus the mode consists of a constant $\Phi$ field and zero velocity field, consistent with geostrophic balance. The secondary branch mode, however, has $\Phi / \mu \rightarrow 0$ as $|\mathbf{k}| \rightarrow 0$, and so has non-zero velocity but zero geopotential perturbation in the limit. Since the $a_{j}$ all tend to 1 , by (41) the three velocity components $u_{1}, u_{2}, u_{3}$ are all constant and equal in value. The velocity field is therefore like that depicted in Fig. 12. The velocity field clearly has small scale structure, even though $\mathbf{k}=\mathbf{0}$. This is best illustrated by the vorticity field, defined at the grid vertices, and indicated by the open and filled circles. This vorticity pattern is consistent with that of a mode of large wavenumber; indeed there is some ambiguity as several different wave vectors are possible, the most obvious being $\mathbf{k} d= \pm(0,4 \pi / \sqrt{3}), \mathbf{k} d= \pm(-2 \pi,-2 \pi / \sqrt{3})$, or $\mathbf{k} d= \pm(2 \pi,-2 \pi / \sqrt{3})$, that is, with wave crests aligned with rows of filled circles in Fig. 12 in one of three possible orientations, parallel to $\hat{\mathbf{x}}_{1}, \hat{\mathbf{x}}_{2}$ or $\hat{\mathbf{x}}_{3}$.

Similar arguments hold for wave vectors other than zero on the secondary 


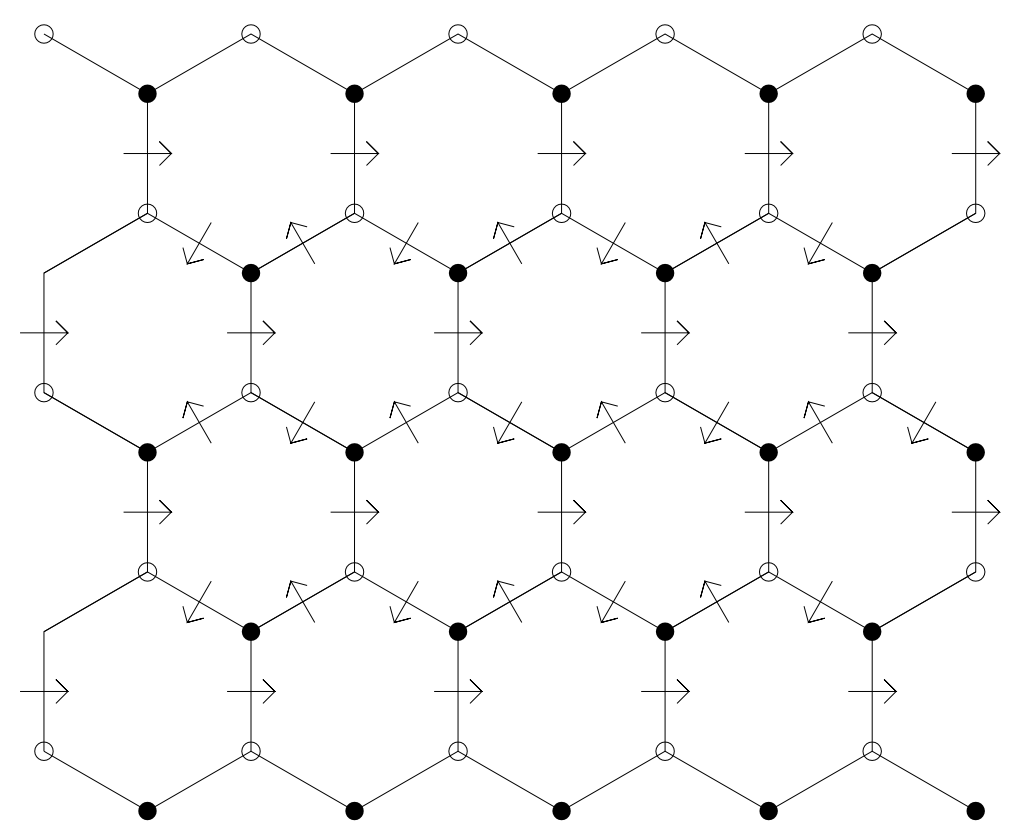

Fig. 12. Schematic showing the small scale structure of the secondary branch Rossby mode with $\mathbf{k}=\mathbf{0}$. Filled circles indicate positive vorticity; open circles indicate negative vorticity.

Rossby mode branch. Thus, in terms of their vorticity structures these modes are plausible approximations to small-scale modes with wave vectors outside the hexagonal region depicted in Figs. 2-5 and 7-8.

We now need to determine whether they are good approximations in terms of their frequencies. For a mode with positive $k$, a negative value of $\omega$ corresponds to westward propagation. The secondary branch Rossby modes have positive $\omega>0$; we can interpret this as westward propagation only if we assume that they represent small scale modes with $k<0$. This suggests we might be able to interpret the secondary branch Rossby mode proportional to $\exp (\mathbf{i k} \cdot \mathbf{x})$ as representing a small-scale mode of aliased wave vector $\mathbf{k}+\mathbf{k}^{\prime}$ where $\mathbf{k}^{\prime} d=(-2 \pi,-2 \pi / \sqrt{3})$. The upper panels of Figs. 9, 10 and 11 show the continuous quasigeostrophic $\beta$-plane Rossby mode frequency at these aliased wave vectors (diamonds) for comparison with the corresponding discrete secondary branch Rossby mode frequencies (plus and cross symbols). For schemes (i) and (ii) it is clear that the discrete Rossby mode frequencies vastly underestimate the continuous Rossby mode frequencies, by at least an order of magnitude and often much more. (Other choices for $\mathbf{k}^{\prime}$ do not improve this conclusion.) Moreover, for scheme (ii) the frequency does not vary monotonically with $l$, implying that group velocity will have the wrong sign for part of the spectrum. Thus, although the hexagonal C-grid is capable of resolving extra small-scale vorticity features, these features will propagate much too slowly, i.e. be excessively passive, and so cannot be expected to contribute additional accuracy to numerical solutions of the governing equations. For scheme (iii) the situation is only slightly better. About one third of the secondary Rossby 
mode branch has frequencies within a factor 10 of the continuous frequencies for aliased wave vectors; however, the discrete frequencies are still too small by at least a factor 6 .

\section{Conclusions}

For a hexagonal C-grid discretization of the $f$-plane shallow water equations, the spurious non-zero frequencies for geostrophic modes noted by previous authors can be avoided by appropriate averaging of the Coriolis terms (27)(29). Three extensions of this averaging that conserve energy in the case of spatially varying $f$ have been presented (section 3 ).

As noted by previous authors, the discrete solution has two Rossby mode branches, giving the same total number of Rossby modes as inertio-gravity modes; this may be contrasted with the case of a quadrilateral C-grid which gives a single Rossby mode branch and only half as many Rossby modes as inertio-gravity modes. A heuristic argument for why this occurs is as follows. On a $\mathrm{C}$-grid the divergence is naturally calculated at $\Phi$ points, while vorticity is naturally calculated at the grid vertices. For both the quadrilateral and hexagonal C-grids, the number of Rossby modes is equal to the number of vorticity degrees of freedom, while the number of inertio-gravity modes is equal to the number of mass plus divergence degrees of freedom. (As an aside, the same reasoning applies on a triangular C-grid, showing that the triangular C-grid supports five times as many inertio-gravity modes as Rossby modes; this might be considered a disadvantage of the triangular C-grid if the Rossby modes are of the greatest physical interest.)

Another view of how the extra Rossby mode branch arises on the hexagonal $\mathrm{C}$-grid is as follows. For the continuous equations, quasigeostrophic theory isolates the Rossby modes by assuming geostrophic balance at leading order, thereby reducing the number of degrees of freedom at each point from three to one. Similar reasoning applies on the quadrilateral C-grid. On the hexagonal C-grid, however, the geostrophic velocity is not uniquely determined by the $\Phi$ field; the ambiguity is expressed mathematically by the field $\mu$ in (41). Thus the quasigeostrophic theory has two degrees of freedom per grid cell, giving rise to two Rossby mode branches.

A quasigeostrophic $\beta$-plane analysis has been carried out (section 4) to determine the discrete dispersion relation for both Rossby mode branches for the three energy conserving schemes. The results have been compared with a direct numerical calculation of the normal mode frequencies (section 5). The primary branches give good approximations to the Rossby mode frequencies of the continuous governing equations, particularly scheme (i), which is more 
accurate than schemes (ii) and (iii) at large wavenumber.

The hexagonal C-grid has very good isotropy properties. The inertio-gravity mode dispersion relation shows only a very weak dependence on the direction of the wave vector relative to the grid; and the primary Rossby mode branch shows only weak dependence on the orientation of the grid relative to the northward direction. Also, particularly for scheme (i), the primary branch Rossby mode frequency remains quite accurate even near the resolution limit, and does not go to zero for the shortest resolvable east-west scales as it does for a quadrilateral C-grid aligned with the north-south and east-west directions.

The secondary branch Rossby modes (section 6) have vorticity structures resembling smaller scale Rossby modes, suggesting that the extra vorticity degrees of freedom on the hexagonal C-grid do translate into extra resolution for vortical modes. However, there is ambiguity over the wave vector of the continuous equation modes that they represent. Also, the frequencies of the secondary branch modes are much smaller than those of the corresponding modes of the continuous equations. Thus, the additional small-scale features that may be resolved in the vorticity field will be excessively passive, and cannot be expected to contribute additional accuracy to numerical solutions. Moreover, the secondary branch dispersion relations are strongly sensitive to the details of the discretization of the Coriolis terms, and, for any choice of discretization, are strongly sensitive to the orientation of the grid relative to the northward direction.

All numerical models of the atmosphere include some form of dissipation on small scales, whether explicitly specified or inherent in the numerical methods used; it serves several purposes, including providing a sink for the downscale potential enstrophy cascade as well as cleaning up noise generated by dispersion errors and parameterization schemes. Secondary branch Rossby modes would be most strongly damped by such scale-selective dissipation. Therefore, it is possible that in practice their poor dispersion properties would not be an issue. In that case, the good isotropy and accurate behaviour at large wavenumber of the inertio-gravity modes and primary branch Rossby modes might make hexagonal grids competitive with quadrilateral grids in terms of accuracy per unit cost.

The results presented here apply only to regular hexagonal grids in planar geometry. Good behaviour of hexagonal C-grid schemes depends crucially on the appropriate averaging of the Coriolis terms. An important extension of this work, therefore, will be to determine how the averaging operator $\widetilde{(.)}$ must be modified for the slightly distorted hexagons and the pentagons on a spherical geodesic grid. 


\section{References}

[1] Arakawa, A., and Lamb, V. R., 1977: Computational design of the basic dynamical processes of the UCLA general circulation model. Methods in Computational Physics, J. Chang, Ed., Vol 17, Academic Press, 173-265.

[2] Bonaventura, L., and Ringler, T., 2005: Analysis of discrete shallow water models on geodesic delaunay grids with C-type staggering. Mon. Wea. Rev., 133, 2351-2373.

[3] Cullen, M. J. P., 1974: Integration of the primitive equations on a sphere using the finite element method. Quart. J. Roy. Meteorol. Soc., 100, 555-562.

[4] Dobricic, S., 2006: An improved calculation of Coriolis terms on the C grid. Mon. Wea. Rev., 134, 3764-3773.

[5] Dukowicz, J. K., 1995. Mesh effects for Rossby Waves. J. Comput. Phys., 119, 188-194.

[6] Fox-Rabinovitz, M. S., 1996: Computational dispersion properties of 3D staggered grids for a nonhydrostatic anelastic system. Mon. Wea. Rev., 124, 498-510.

[7] Gavrilov, M. B., and Tošić, I. A., 1998. Propagation of the Rossby waves on two dimensional rectangular grids. Meteorol. Atmos. Phys., 68, 119-125.

[8] Giraldo, F. X., 2000: Lagrange-Galerkin methods on spherical geodesic grids: The shallow-water equations. J. Comput. Phys., 160, 336-368.

[9] Heikes, R., and Randall, D. A., 1995: Numerical integration of the shallow-water equations on a twisted icosahedral grid. Part I: Basic design and results of tests. Mon. Wea. Rev., 123, 1862-1887.

[10] Le Roux, D. Y., Staniforth, A., and Lin, C. A., 1998: Finite elements for shallowwater equation ocean models. Mon. Wea. Rev., 126, 1931-1951.

[11] Majewski, D., Liermann, D., Prohl, P., Ritter, B., Buchhold, M., Hanisch, T., Paul, G., and Wergen, W., 2002: The operational global icosahedral-hexagonal gridpoint model GME: Description and high-resolution tests. Mon. Wea. Rev., $130,319-338$.

[12] Masuda, Y., and Ohnishi, H., 1986: An integration scheme of the primitive equation model with an icosahedral-hexagonal grid system and its application to the shallow-water equations. In Short- and Medium-Range Numerical Weather Prediction. Collection of Papers Presented at the WMO/IUGG NWP Symposium, Tokyo, Aug.4-8, 1986. Japan Meteorological Society, 317-326.

[13] Miura, H., and Kimoto, M., 2005: A comparison of grid quality of optimized spherical hexagonal-pentagonal geodesic grids. Mon, Wea, Rev., 133, 2817-2833.

[14] Ničković, S., Gavrilov, M. B., and Tosić, I. A., 2002: Geostrophic adjustment on hexagonal grids. Mon. Wea. Rev., 130, 668-683. 
[15] Popović, J. M., Ničković, S., and Gavrilov, M. B., 1996: Frequency of quasigeostrophic modes on hexagonal grids. Meteorol. Atmos. Phys., 58, 4149.

[16] Randall, D. A., 1994: Geostrophic adjustment and the finite-difference shallowwater equations. Mon. Wea. Rev., 122, 1371-1377.

[17] Ringler, T., Heikes, R., and Randall, D. A., 2000: Modeling the atmospheric general circulation using a spherical geodesic grid. Mon. Wea. Rev., 128, 24712490 .

[18] Ringler, T., and Randall, D., 2002: A potential enstrophy and energy conserving numerical scheme for solution of the shallow-water equations on a spherical geodesic grid. Mon. Wea. Rev., 130, 1397-1410.

[19] Sadourny, R., 1969: Numerical integration of the primitive equations on a spherical grid with hexagonal cells. Proc. WMO/IUGG NWP Symp., Tokyo, Japan, Japan Meteorological Agency, 45-52.

[20] Satoh, M., Matsuno, T., Tomita, H., Nasuno, T., Iga, S., and Miura, H., 2007: Nonhydrostatic Icosahedral Atmospheric Model (NICAM) for global cloud resolving simulations. J. Comput. Phys. in press. doi:10.1016/j.jcp.2007.02.006

[21] Stuhne, G. R., and Peltier, W. R., 1999: New icosahedral grid-point discretizations of the shallow water equations on the sphere. J. Comput. Phys., $148,23-58$.

[22] Thuburn, J., 1997: A PV-based shallow water model on a hexagonal-icosahedral grid. Mon. Wea. Rev., 125, 2328-2347.

[23] Thuburn, J., 2007: Rossby wave propagation on the C-grid. Atmos. Sci. Lett., $8,37-42$.

[24] Thuburn, J., and Staniforth, A., 2004: Conservation and linear Rossby-mode dispersion on the spherical C grid. Mon. Wea. Rev., 132, 641-653.

[25] Torsvik, T., Thiem, Ø., and Bernsten, J., 2005: Stability analysis of geostrophic adjustment on hexagonal grids for regions with variable depth. Mon. Wea. Rev., $133,3335-3344$.

[26] Wajsowicz, R. C., 1986. Free planetary waves in finite-difference numerical models. J. Phys. Oceanogr., 16, 773-789.

[27] Williamson, D., 1968: Integration of the barotropic vorticity equation on a spherical geodesic grid. Tellus, 20, 624-653. 